\title{
Large-scale identification of human genes implicated in epidermal barrier function
}

\author{
Eve Toulza* ${ }^{*}$ Nicolas R Mattiuzzo*, Marie-Florence Galliano*, \\ Nathalie Jonca* ${ }^{*}$, Carole Dossat ${ }^{\dagger}$, Daniel Jacob ${ }^{\ddagger}$, Antoine de Daruvar ${ }^{\ddagger}$, \\ Patrick Wincker ${ }^{\dagger}$, Guy Serre* and Marina Guerrin*
}

\begin{abstract}
Addresses: *UMR 5165 "Epidermis Differentiation and Rheumatoid Autoimmunity", CNRS - Toulouse III University (IFR 30, INSERM - CNRS - Toulouse III University - CHU), allées Jules Guesde, 31073 Toulouse, France. 'Genoscope and CNRS UMR 8030, rue Gaston Crémieux, 91057 Evry, France. ${ }^{\ddagger}$ Centre de Bioinformatique Bordeaux, Université V. Segalen Bordeaux 2, rue Léo Saignat, 33076 Bordeaux Cedex, France.
\end{abstract}

Correspondence: Marina Guerrin. Email: mweber@udear.cnrs.fr

Published: II June 2007

Genome Biology 2007, 8:RI07 (doi:10.1 186/gb-2007-8-6-r107)

The electronic version of this article is the complete one and can be found online at http://genomebiology.com/2007/8/6/R I07
Received: I March 2007

Revised: 24 May 2007

Accepted: II June 2007

(C) 2007 Toulza et al.; licensee BioMed Central Ltd.

This is an open access article distributed under the terms of the Creative Commons Attribution License (http://creativecommons.org/licenses/by/2.0), which permits unrestricted use, distribution, and reproduction in any medium, provided the original work is properly cited.

\begin{abstract}
Background: During epidermal differentiation, keratinocytes progressing through the suprabasal layers undergo complex and tightly regulated biochemical modifications leading to cornification and desquamation. The last living cells, the granular keratinocytes (GKs), produce almost all of the proteins and lipids required for the protective barrier function before their programmed cell death gives rise to corneocytes. We present here the first analysis of the transcriptome of human GKs, purified from healthy epidermis by an original approach.

Results: Using the ORESTES method, 22,585 expressed sequence tags (ESTs) were produced that matched 3,387 genes. Despite normalization provided by this method (mean 4.6 ORESTES per gene), some highly transcribed genes, including that encoding dermokine, were overrepresented. About 330 expressed genes displayed less than 100 ESTs in UniGene clusters and are most likely to be specific for GKs and potentially involved in barrier function. This hypothesis was tested by comparing the relative expression of 73 genes in the basal and granular layers of epidermis by quantitative RT-PCR. Among these, 33 were identified as new, highly specific markers of GKs, including those encoding a protease, protease inhibitors and proteins involved in lipid metabolism and transport. We identified filaggrin 2 (also called ifapsoriasin), a poorly characterized member of the epidermal differentiation complex, as well as three new lipase genes clustered with paralogous genes on chromosome 10q23.3I. A new gene of unknown function, Clorf8I, is specifically disrupted in the human genome by a frameshift mutation.
\end{abstract}

Conclusion: These data increase the present knowledge of genes responsible for the formation of the skin barrier and suggest new candidates for genodermatoses of unknown origin. 


\section{Background}

High-throughput genomic projects focusing on the identification of cell- and tissue-specific transcriptomes are expected to uncover fundamental insights into biological processes. Particularly intriguing are genes in sequenced genomes that remain hypothetical and/or poorly represented in expressed sequence databases, and whose functions in health and disease remain unknown. Some of these are most probably implicated in organ-specific functions. Their characterization is essential to complete the annotation of sequenced genomes and is expected to contribute to advances in physiology and pathology. In order to achieve such goals, transcriptome studies on tissues rather than cultured cells, and eventually on a single cell type at a precise differentiation step are more likely to provide new information.

The epidermis is a highly specialized tissue mainly dedicated to the establishment of a barrier that restricts both water loss from the body and ingress of pathogens. The barrier function of the epidermis is known to involve the expression of numerous tissue-specific genes, most of which are specifically expressed in the late steps of keratinocyte differentiation. In order to establish and constantly maintain this barrier, keratinocytes undergo a complex, highly organized and tightly controlled differentiation program leading to cornification and finally to desquamation. During this process, cells migrate from the basal, proliferative layer to the surface, where they form the cornified layer (stratum corneum). According to the current model of skin epithelial maintenance, basal keratinocytes encompass a heterogeneous cell population that includes slow-cycling stem cells [1]. These stem cells give rise to transiently amplifying keratinocytes that constitute most of the basal layer. They divide only a few times and finally move upward while differentiating to form the spinous layer. The proliferating compartment is characterized by the specific expression of cell cycle regulators and integrin family members responsible for the attachment of the epidermis to the basement membrane. Growth arrested keratinocytes undergo differentiation, mainly characterized by a shift in cytokeratin expression from $\mathrm{KRT}_{5}$ (keratin 5 ) and KRT14 in the basal layer to KRT1 and KRT10 in suprabasal layers. As differentiation progresses, keratinocytes from the spinous layers progressively express a small number of specific differentiation markers, like involucrin. However, the differentiation program culminates in the granular layer, where keratinocytes express more than 30 epidermis-specific proteins, including proteins that are stored in cytosolic granules characteristic of granular keratinocytes (GKs). These proteins include well known components of the cornified layer, like loricrin and elafin, but also recently identified ones, such as keratinocyte differentiation associated protein (KDAP), hornerin, suprabasin, keratinocyte proline rich protein (hKPRP), and so on [2-5].

GKs undergo a special programmed cell death, called cornification, which gives rise to corneocytes that no longer exhibit transcriptional or translational activity and are devoid of organelles. Rather, their intracellullar content consists of a homogeneous matrix composed mainly of covalently linked keratins. The cornified envelope, a highly specialized insoluble structure, encapsulates corneocytes in place of their plasma membrane (see Kalinin et al. [6] for a recent review). The lipid-enriched extracellular matrix, which subserves the barrier, is produced by a highly active lipid factory mainly operative in the granular layer and comprises secretory organelles named the epidermal lamellar bodies [7]. In addition to the provision of lipids for the barrier, lamellar bodies deliver a large number of proteins, including lipid-processing enzymes, proteases and anti-proteases that regulate desquamation, antimicrobial peptides and corneodesmosin, an adhesive protein secondarily located in the external face of the desmosomes, as they turn into corneodesmosomes [8]. Therefore, the components of the stratum corneum, responsible for most of the protective cutaneous functions, are produced by GKs.

Transcriptome studies of selected cell types of the human epidermis are expected to contribute to the elucidation of the mechanisms responsible for barrier function. They will also shed further light on the causes of monogenic genodermatoses and the pathomechanisms of common complex skin disorders like psoriasis. However, present knowledge on the gene repertoire expressed by keratinocytes remains largely fragmentary. Among the approximately eight million human expressed sequence tags (ESTs) from the dbEST division of the GenBank database, only 1,210 are annotated as originating from the epidermis, although these are, in fact, derived from cultured keratinocytes, which do not fully recapitulate the complex in vivo differentiation program. In this article, we describe the results of a large-scale cDNA sequencing project on GKs of healthy human skin, purified by a new method. In order to characterize genes expressed at a low level and to avoid the repetitive sequencing of highly expressed ones, we used the ORESTES (open reading frame EST) method to prepare a large series of small size cDNA libraries using arbitrarily chosen primers for reverse transcription (RT) and PCR amplification [9]. The sequencing of about 25,000 clones has produced a list of 3,387 genes expressed by GKs. Some of them, analyzed by quantitative RT-PCR, were shown to be expressed in a cell-specific manner. This effort resulted in a large number of novel candidate genes of importance for the epidermis barrier function and the etiology of genodermatoses.

\section{Results \\ Purification of human granular keratinocytes}

As a first step in this transcriptome project we devised a method to purify GKs. Iterative incubations of pieces of human epidermis with trypsin were performed to give three suspended cell fractions (hereafter named $\mathrm{T}_{1}-\mathrm{T}_{3}$ ) and finally to isolate cells attached to the stratum corneum ( $\mathrm{T}_{4}$ fraction). 
Table I

Expression ratios for KRTI4 and KLK7 as measured by real-time PCR from four independent samples

\begin{tabular}{lcccc}
\hline & \multicolumn{5}{c}{ Sample no. } \\
\cline { 2 - 5 } Expression ratio & 1 & 2 & 3 & 4 \\
\hline KRTI4 (TI/T4) & 7.5 & 5.9 & 25 & 13.6 \\
SCCE/KLK7 (T4/TI) & 164 & 189 & 120 & 54 \\
\hline
\end{tabular}

Morphological analyses revealed that after three treatments, residual epidermal fragments were mostly composed of corneocytes and GKs (Figure 1). Quantitative real-time PCR was performed to quantify the enrichment in GKs. To first select a reference gene for normalization, the relative expression of eight housekeeping genes (GAPDH, SOD1, ACTB, B2M, $H P R T 1, H M B S, T B P$ and $U B C$ ) in each cell fraction (T1-T4) was analyzed using GeNorm [10]. In agreement with previous data [11], beta-2-microglobulin (B2M) appeared to be stably expressed during epidermis differentiation, and was thus chosen for normalization. In addition, we used the lectin Galectin-7 ( $L S G A L 7$ ), which was previously shown by in situ hybridization to be equally expressed in all epidermal layers [12]. BPAG2 (bullous pemphigoid antigen 2) or KRT14, and $K L K 7$ (kallikrein 7, also called stratum corneum chymotryptic enzyme (SCCE)) were selected as specific for the basal layer or the GKs, respectively [13,14]. For four cell fractionations from different individuals, the mean $\mathrm{T} 1 / \mathrm{T} 4$ expression ratio of KRT14 was 13 , whereas the mean $\mathrm{T} 4 / \mathrm{T} 1$ expression ratio of SCCE was approximately 130 (Table 1 ). The KRT14 ratio might be indicative of a slight contamination of the $\mathrm{T} 4$ fraction with basal keratinocytes. Nevertheless, the large SCCE ratio indicates that very few, if any, GKs were present in the T1 fraction. From this, we concluded that the T4 fraction was highly enriched in GKs and thus suitable for a large-scale study of their transcriptome.

\section{An ORESTES dataset from human granular keratinocytes}

PolyA + RNA was extracted from the $\mathrm{T} 4$ fraction from individual 3 (Table 1) and used to generate cDNA mini-libraries using the ORESTES method [9]. This sample was chosen as it presents the highest $\mathrm{T} 1 / \mathrm{T}_{4}$ expression ratio for the KRT14 gene, suggesting a low contamination of the $\mathrm{T} 4$ fraction by basal keratinocytes. This method uses arbitrarily chosen primers for reverse transcription and PCR amplification. The successful amplification of a mRNA thus depends primarily on partial sequence homology with the primer, rather than on its abundance. This, and the elimination of cDNA preparations that display prominent bands on gels (indicative of the selective amplification of particular mRNAs), results in a normalization process and allows the detection of rare transcripts. We constructed 150 cDNA libraries with different primers, the analysis of 100-200 clones from each leading to the production of 22,585 sequences (Figure 2a). Among these, 1,453 (approximately 6\%) corresponded to empty plasmids or uninformative sequences, 377 (1.7\%) were of bacterial origin, and 2,303 (10\%) matched the human mitochondrial genome. Despite two rounds of polyA ${ }^{+}$RNA purification, 1,859 sequences $(8.2 \%)$ arose from ribosomal RNA. In addition, 187 sequences corresponded to unspliced intergenic DNA and may reflect spurious transcriptional activity. The remaining 16,591 sequences (73\%) matched known or predicted transcribed regions, of which $62 \%$ aligned with the human genome in several blocks, and thus corresponded to spliced transcripts. After clustering, we observed the transcription of 3,387 genes by GKs. Additionally, 23 sequences matched overlapping exons belonging to two genes transcribed in opposite orientations and thus could not be attributed to a single gene.

The normalization ability of the ORESTES method was examined by classifying genes according to the number of matching sequences in the dataset (Figure 2b). Half of the genes were represented by a unique sequence and $76.3 \%$ by three or less sequences, thus showing an acceptable level of normalization, with a mean of 4.6 ORESTES per gene. However, the ORESTES method only partially compensates for transcript abundance, as several genes were represented by a large ORESTES number. In these cases, we examined the number of sequences in the corresponding UniGene clusters, a rough measure of gene expression level. This revealed two situations: first, the gene is strongly expressed in many cell types including GKs (a high number of both ORESTES and UniGene entries); and second, the gene is particularly expressed in GKs (a high number of ORESTES, but low number of UniGene entries). The first category mainly includes housekeeping genes from the translation machinery (for example, RPS8, EEF1A1, RPL3, RPL7A, RPL28; Table 2). The second category contains genes previously described as implicated in epidermis barrier function (for example, KRT1, DMKN, LEP7, FLG, $K R T 2 A, S P R R 2 E, C A S P 14, C D S N$, hKRP, SBSN) and, interestingly, new candidates for this function (TSPAN5, DUOX2, TMEM14C, SERPINA12, SLC22A5, FLG2, C7orf24). Dermokine $(D M K N)$, represented by 217 ORESTES, was shown to be selectively transcribed in mouse GKs by highthroughput in situ hybridization [15] and signal sequence trap [16] screens. The present ORESTES dataset allowed us to describe 13 novel human DMKN splicing isoforms with distinct subcellular locations and expression patterns [17]. 


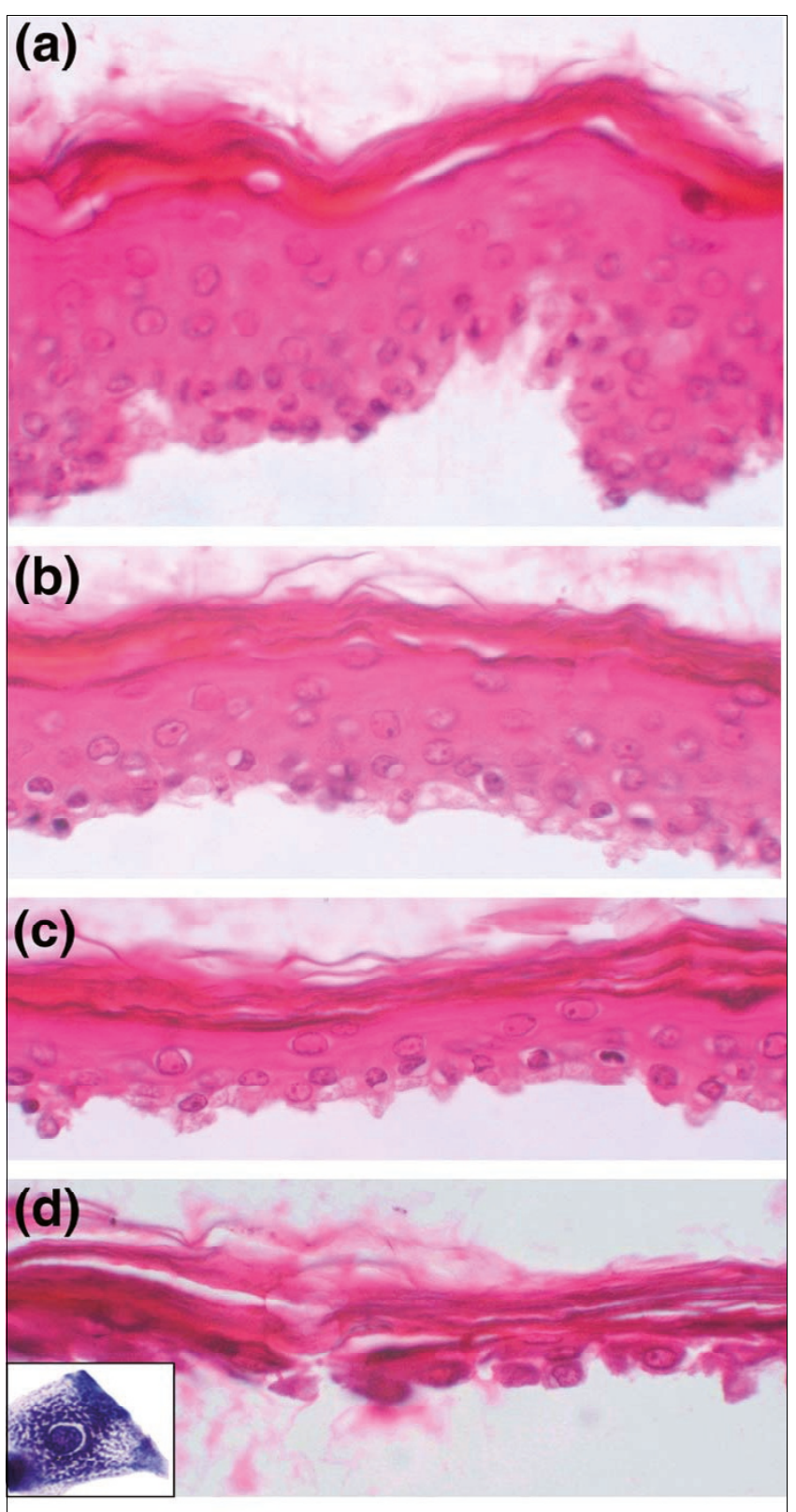

Figure I

Histological analysis of epidermis samples. (a) Hematoxylin-eosin stained sections of entire epidermis after thermolysin incubation and removal of the dermis. (b,c,d) Epidermis fragments remaining after the first, second, and third trypsin incubation, respectively. Fragments shown in (d) are mainly composed of GKs attached to the cornified layer and constitute the T4 fraction. Inset: higher magnification showing the characteristic cytological aspect of a GK with cytoplasmic keratohyalin granules.

The ORESTES dataset was aligned with the human genome using BLAT [18]. The BLAT results were used to write a custom track that allows the visualization of the position of a particular ORESTE relative to other annotations such as RefSeq genes, vertebrate orthologues, single nucleotide polymorphisms, microarray expression data, and so on, and is freely available online [19]. A screen copy of a UCSC Genome
Browser window showing the ORESTES obtained for the C1orf81 gene is presented as an example (Additional data file 1). Indeed, this gene was characterized and a cDNA (DQ983818) was cloned for the first time in this study (see below). Our dataset includes the 16,591 ESTs matching known or predicted transcribed regions. These sequences have also been deposited in public databases (GenBank:EL593304-EL595248, GenBank:CU442764CU457374).

\section{Poorly represented genes in expressed sequence databases}

As few sequencing projects from human epidermis have been performed so far (relative to other organs), genes expressed during the late steps of epidermis differentiation are poorly represented in sequence databases. Among the 3,375 genes from our set, 330 (10\%) corresponded to UniGene clusters containing less than $100 \mathrm{mRNA} / \mathrm{EST}$ sequences, and were thus good candidates for epidermis late-expressed genes. These were subdivided into five classes. The first one contains all the genes (50) already known to be specifically expressed in the suprabasal layers (Table 3). This confirms that lateexpressed genes are poorly represented in EST databases. The second class consisted of 31 genes with known or inferred functions that were previously known as mainly expressed in a specific tissue different from epidermis (Table 4). We suggest that some of them might play a specific role in epidermal differentiation. This could be the case for SERPINA12, $D U O X 2$, and, to a lesser extent, $C A S Z 1$, which are represented by a large ORESTES number. We also suspect that $C L D N 23$ might play an important role in GKs, since claudin-based tight junctions in the granular layer contribute to barrier function of the epidermis [20]. Accordingly, claudin-1-deficient mice display a lethal defect in skin permeability [21]. The third class gathered 32 uncharacterized paralogues of known genes (Table 5). The fourth class was composed of 105 genes that remain hypothetical and about which nothing is known regarding their normal function or disease relevance (Table 6). The fifth class contained genes that are expressed, most probably at low levels, in numerous tissues, but whose epidermal expression is, to the best of our knowledge, described here for the first time (Additional data file 2). Several genes from these five classes were selected to quantify their expression in the course of epidermal differentiation by real-time PCR (see below).

\section{Expressed retrogenes and pseudogenes}

Pseudogenes generally correspond to retrocopies with many disruptions in their open reading frame (ORF). However, it is now recognized that a large number of retrocopies are transcribed and can encode functional proteins [22]. Among the top 50 transcribed retrocopies reported by these authors, 11 were detected in GKs by the ORESTES method. Among these, calmodulin-like $3(C A L M L 3)$ was previously shown to be specific for keratinocyte terminal differentiation [23]. We identified two other expressed retrogenes corresponding to the 


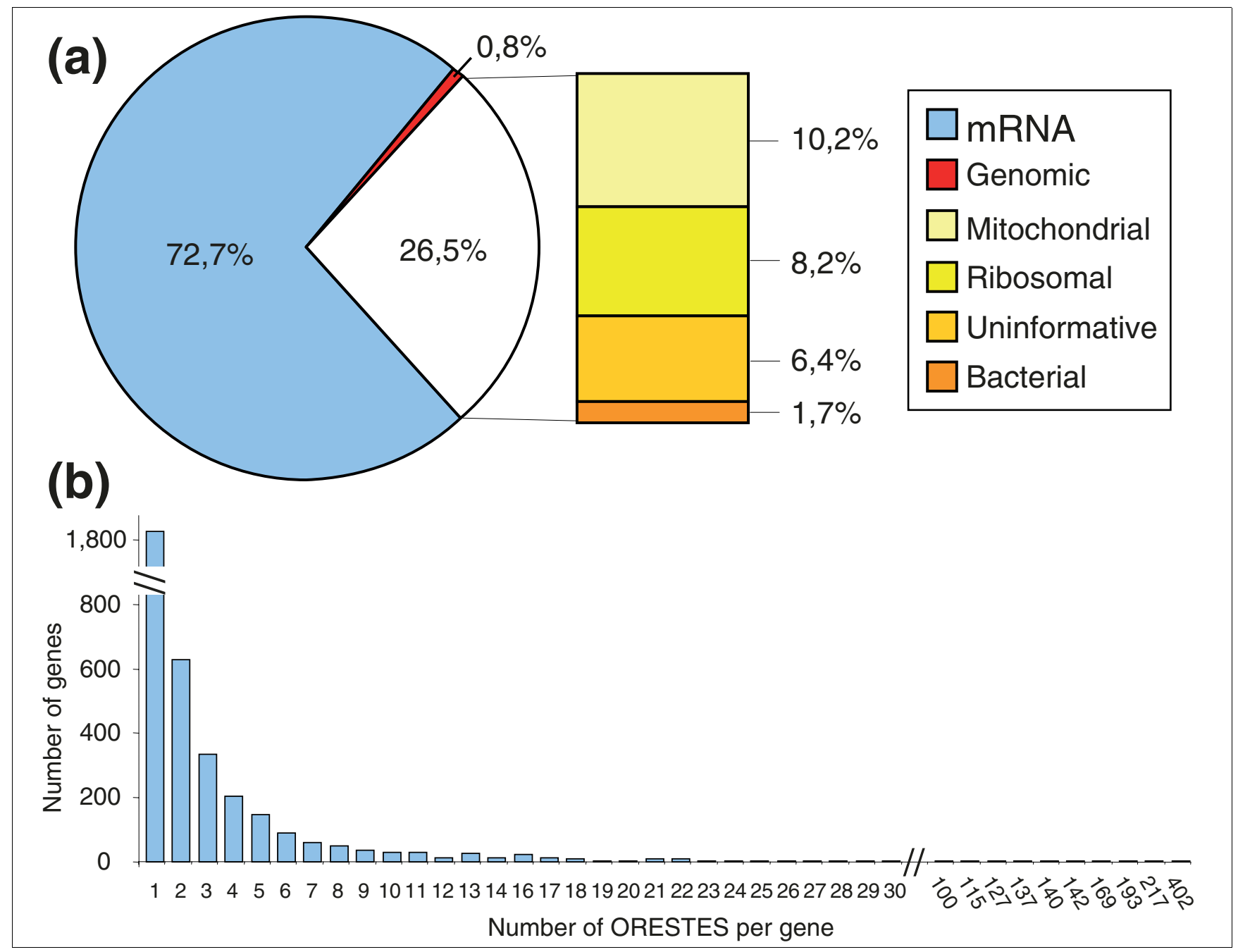

Figure 2

Analysis of the ORESTES dataset from GKs. (a) Pie graph of the 22,585 sequences obtained from the T4 fraction enriched in GKs. The treatment of the mRNA samples with DNAse resulted in minimal contamination with genomic sequences. Despite two rounds of polyA+ mRNA purification, rRNA sequences still represent approximately $8 \%$ of the dataset. (b) Histogram showing the number of ORESTES at each level of redundancy. The vast majority of genes are represented by less than five ORESTES, illustrating the normalization capability of that method. However, a small number of genes are represented by a large number of ORESTES (up to 402).

retrotransposition of the cutaneous T-cell lymphoma associated antigen 5 (CTAGE5), and CCR4-NOT transcription complex, subunit 6-like (CNOT6L). These genes can be considered as 'intact', that is, they show no disablements such as premature stop codons or frameshift mutations when compared to the ORF of their parental genes. Of note, the CNOT6L retrogene is specific for hominoids (Additional data file 3), while the CTAGE5 retrogene is specific for primates (data not shown).

Moreover, six unspliced ORESTES correspond to a part of intron 8 of the $P P P 2 R 5 A$ gene, and include the small nucleolar RNA (snoRNA) U98b sequence. The snoRNAs are nonprotein-coding RNAs that guide the 2'O-ribose methylation (C/D box snoRNAs) or the pseudouridylation (H/ACA box snoRNAs) of ribosomal RNAs, and are generally processed from introns of RNA polymerase II transcripts [24]. Interestingly, the U98b snoRNA is a primate-specific retroposon of the $A C A 16$ snoRNA hosted by the PNAS-123 gene [25]. We thus suggest that the ORESTES from the $P P P 2 R 5 A$ gene correspond to a precursor form of the U98b snoRNA, and that snoRNA retroposons can indeed be expressed when located in an intron of a new host gene in the sense orientation. Therefore, our ORESTES dataset included transcripts from retrogenes, originating either from spliced pre-mRNAs or from an intron-encoded snoRNA gene.

\section{Non-protein-coding genes}

We obtained two long spliced ORESTES highly similar to the BCo70486 mRNA form of the GAS5 gene, a non-protein-coding gene that belongs to the 'growth arrest specific' family but is disrupted in its ORF by a premature stop codon. The GAS5 
Table 2

\begin{tabular}{|c|c|c|c|}
\hline No. of ORESTES & Gene symbol & No. of UniGene ESTs & Full name (alias) \\
\hline \multicolumn{4}{|c|}{ Ubiquitously expressed genes with a high number of UniGene ESTs } \\
\hline 142 & RPS8 & 3,382 & Ribosomal protein S8 \\
\hline 115 & EEFIAI & 29,374 & Eukaryotic translation elongation factor I alpha I \\
\hline 77 & $H L A-B$ & 4,536 & Major histocompatibility complex, class I, B \\
\hline 71 & RPL3 & $|1,56|$ & Ribosomal protein L3 \\
\hline 62 & NCL & 2,970 & Nucleolin \\
\hline 55 & RPL28 & 2,394 & Ribosomal protein L28 \\
\hline 55 & RPL7A & 5,864 & Ribosomal protein $17 \mathrm{a}$ \\
\hline 51 & RPSA & 5,623 & Ribosomal protein SA \\
\hline 50 & PABPCI & 4,385 & Poly(A) binding protein, cytoplasmic I \\
\hline 34 & RPSI8 & 2,292 & Ribosomal protein SI8 \\
\hline \multicolumn{4}{|c|}{ Known epidermis specific genes } \\
\hline 402 & KRTI & 134 & Keratin I \\
\hline 217 & DMKN & 275 & Dermokine \\
\hline 140 & LEP7 & 5 & Late envelope protein 7 (xp32) \\
\hline 100 & FLG & 5 & Filaggrin \\
\hline 71 & KRT2A & 12 & Keratin $2 \mathrm{~A}$ \\
\hline 62 & SPRR2E & 36 & Small proline-rich protein $2 \mathrm{E}$ \\
\hline 61 & CASPI 4 & 19 & Caspase 14 \\
\hline 59 & $\operatorname{CDSN}$ & 91 & Corneodesmosin \\
\hline 56 & hKPRP & 7 & Human keratinocyte proline rich protein \\
\hline 54 & PKPI & 263 & Plakophilin I \\
\hline 32 & SBSN & 49 & Suprabasin \\
\hline 30 & DSGI & 61 & Desmoglein I \\
\hline \multicolumn{4}{|c|}{ Genes with unknown function } \\
\hline 193 & TSPAN5 & 526 & Tetraspanin 5 \\
\hline 127 & DUOX2 & 64 & Dual oxidase 2 \\
\hline 99 & TMEMI4C & 476 & Transmembrane protein $14 \mathrm{C}$ \\
\hline 99 & SERPINA I 2 & 11 & Serpin peptidase inhibitor, clade A, member 12 \\
\hline 66 & SLC22A5 & 142 & Solute carrier family 22, member 5 \\
\hline 56 & FLG2 & 10 & Filaggrin 2 (ifapsoriasin) \\
\hline 41 & C7orf24 & 309 & Chromosome 7 open reading frame 24 \\
\hline
\end{tabular}

gene is the host gene for $10 \mathrm{C} / \mathrm{D}$ box snoRNAs [26]. Other snoRNA host genes included in our ORESTES dataset are RPS11, RPS12, RPL1O and EIF4A1. In certain cases, ORESTES contain the snoRNA sequence (U39B in RPS11, mgU6-77 in EIF4A1, U70 in RPL1O), and probably correspond to alternative splicing forms of the host gene mRNA, with intron retention.

We furthermore obtained sequences for long, non-proteincoding transcripts. Metastasis associated lung adenocarcinoma transcript 1, (MALAT-1, 22 ORESTES) is a conserved long non-protein-coding RNA (>8,000 nucleotides (nt)) of unknown function that is highly expressed in numerous healthy organs and overexpressed in metastatic non-small cell lung carcinomas [27]. Close to MALAT-1 on 11q13.1, trophoblast-derived noncoding RNA (TncRNA, 44
ORESTES) is a 481 nucleotide (nt), non-protein-coding RNA involved in trophoblastic major histocompatibility complex suppression by inhibiting class II transactivator (CIITA) transcription [28]. $\mathrm{H}_{19}$ is a non-protein-coding, maternally imprinted mRNA (two spliced ORESTES) [29] that is highly transcribed in extraembryonic and fetal tissues, as well as in adult skeletal muscle. It has been shown that $\mathrm{H} 19$ is involved in the genomic imprinting of the insulin-like growth factor 2 (IGF2) gene [30]. Moreover, IGF2 is expressed throughout the epidermis [31] and its overexpression increases the thickness of the epidermis and the proportion of dividing cells in the basal layer [32]. We suggest that $H 19$ could participate in the regulation of IGF2 transcription by maintaining the genomic imprinting of its promoter in adult epidermis. In addition to numerous protein-coding genes, we thus detected several non-protein-coding RNAs whose expression in the 
Table 3

\section{Genes with less than 100 UniGene ESTs encoding known GK expressed proteins}

\begin{tabular}{|c|c|c|c|}
\hline No. of ORESTES & Gene symbol & No. of UniGene ESTs & Full name (alias) \\
\hline 2 & LCEIF & I & Late cornified envelope IF \\
\hline 4 & LCE2C & 1 & Late cornified envelope $2 \mathrm{C}$ \\
\hline I & Clorf46 & 2 & Chromosome I open reading frame 46 (xp 33) \\
\hline I & LCE2A & 3 & Late cornified envelope $2 \mathrm{~A}$ \\
\hline 6 & LCE5A & 3 & Late cornified envelope $5 \mathrm{~A}$ \\
\hline 11 & LCEIA & 3 & Late cornified envelope IA \\
\hline 2 & PGLYRP3 & 5 & Peptidoglycan recognition protein 3 \\
\hline 5 & LCEIC & 5 & Late cornified envelope IC \\
\hline 100 & FLG & 5 & Filaggrin \\
\hline 140 & LEP7 & 5 & Late envelope protein 7 \\
\hline 2 & RPTN & 6 & Repetin \\
\hline I & $L C E 2 B$ & 7 & Late cornified envelope $2 B$ \\
\hline 56 & $h K P R P$ & 7 & Human keratinocyte proline rich protein \\
\hline 9 & $L O R$ & 11 & Loricrin \\
\hline 71 & KRT2A & 12 & Keratin $2 \mathrm{~A}$ \\
\hline 2 & Clorf42 & 15 & Chromosome I open reading frame 42 (NICE-I) \\
\hline 5 & TGM5 & 15 & Transglutaminase 5 \\
\hline 13 & DSCI & 17 & Desmocollin I \\
\hline 16 & $K R T I B$ & 18 & Keratin IB \\
\hline 61 & CASPI 4 & 19 & Caspase 14 \\
\hline I & CNFN & 20 & Cornifelin \\
\hline 4 & CALML5 & 25 & Calmodulin-like 5 \\
\hline I & ALOXE3 & 28 & Arachidonate lipoxygenase 3 \\
\hline 8 & $A L O X I 2 B$ & 30 & Arachidonate 12-lipoxygenase, I2R type \\
\hline 62 & SPRR2E & 36 & Small proline-rich protein $2 \mathrm{E}$ \\
\hline 17 & $I V L$ & 38 & Involucrin \\
\hline 2 & EPPKI & 42 & Epiplakin I \\
\hline 5 & POU2F3 & 45 & POU domain, class 2, transcription factor 3 (oct- II) \\
\hline 4 & ICHTHYIN & 48 & Ichthyin \\
\hline 32 & SBSN & 49 & Suprabasin \\
\hline 2 & $K L K 8$ & 53 & Kallikrein 8 (neuropsin/ovasin) \\
\hline 4 & TGM3 & 54 & Transglutaminase 3 \\
\hline I & $A B C A / 2$ & 55 & ATP-binding cassette, sub-family $A(A B C I)$, member 12 \\
\hline 3 & PADII & 56 & Peptidylarginine deiminase, type I \\
\hline 30 & DSGI & 61 & Desmoglein I \\
\hline 2 & GJB3 & 65 & Gap junction protein, beta 3 (connexin $3 \mathrm{I}$ ) \\
\hline 1 & CALML3 & 68 & Calmodulin-like 3 \\
\hline 13 & SASpase & 69 & Skin aspartic protease \\
\hline 15 & $K L K 7 / S C C E$ & 69 & Kallikrein 7 (Stratum corneum chymotrypticenzyme) \\
\hline 6 & $A 2 M L I$ & 76 & Alpha-2-macroglobulin-like I \\
\hline I & CST6 & 78 & Cystatin E/M \\
\hline I & SULT2BI & 80 & Sulfotransferase family, cytosolic, 2B, member I \\
\hline 2 & $K L K I I$ & 83 & Kallikrein II \\
\hline 3 & $H A L$ & 86 & Histidine ammonia-lyase (histidase) \\
\hline 14 & EVPL & 91 & Envoplakin \\
\hline 59 & CDSN & 91 & Corneodesmosin \\
\hline 3 & $P D Z K I I P I$ & 92 & PDZKI interacting protein I \\
\hline 4 & TGMI & 92 & Transglutaminase I \\
\hline 2 & SERPINB8 & 99 & Serpin peptidase inhibitor, clade B, member 8 \\
\hline 20 & SCEL & 99 & Sciellin \\
\hline
\end{tabular}


Table 4

Genes with 100 or less UniGene ESTs, known as mainly expressed in a specific tissue different from epidermis

\begin{tabular}{|c|c|c|c|c|}
\hline No. of ORESTES & Gene symbol & No. of UniGene ESTs & Full name & Main specificity \\
\hline 99 & SERPINA I 2 & 11 & Serpin peptidase inhibitor, clade A, member 12 & Adipocytes \\
\hline 1 & BSND & 12 & Bartter syndrome, infantile, with sensorineural deafness & Kidney and inner ear \\
\hline 1 & OPNILW & 16 & Opsin I, long-wave-sensitive & Eye \\
\hline 5 & GRIN2 & 16 & G-protein-regulated inducer of neurite outgrowth & Brain \\
\hline 2 & ILIRL2 & 24 & Interleukin I receptor-like 2 & Neurons \\
\hline 13 & $L C T L$ & 25 & Lactase-like & Kidney \\
\hline 1 & PPEF2 & 31 & Protein phosphatase, EF-hand calcium binding domain 2 & Retina \\
\hline 2 & SLC6A3 & 34 & Solute carrier family 6 , member 3 & Neuron \\
\hline 3 & CDC42BPG & 41 & CDC42 binding protein kinase gamma & Heart and skeletal muscle \\
\hline 4 & GPR75 & 41 & G protein-coupled receptor 75 & Retina \\
\hline 1 & OTXI & 45 & Orthodenticle homolog I & Neurons \\
\hline 1 & $K 5 B$ & 46 & Keratin $5 b$ & Tongue \\
\hline I & $T B X 15$ & 46 & T-box 15 & Embryo \\
\hline 3 & TMPRSS5 & 53 & Transmembrane protease, serine 5 (spinesin) & Spinal chord \\
\hline 3 & LEAP-2 & 53 & Liver-expressed antimicrobial peptide 2 & Liver \\
\hline 1 & $B M P 8 B$ & 56 & Bone morphogenetic protein $8 \mathrm{~B}$ & Embryo \\
\hline 1 & PTGFR & 59 & Prostaglandin F receptor & Uterus \\
\hline 2 & TEC & 59 & Tec protein tyrosine kinase & Hematopoietic cells \\
\hline 3 & SLC5AI & 60 & Solute carrier family 5 , member I & Intestine and kidney \\
\hline 20 & CASZI & 61 & Castor homolog I, zinc finger & Mesenchyme \\
\hline 2 & $K C N J I 2$ & 62 & Potassium inwardly rectifying channel, subfamily J, 12 & Heart \\
\hline 1 & PII & 64 & 26 serine protease & Placenta \\
\hline 14 & SERPINB7 & 64 & Serpin peptidase inhibitor, clade B, member 7 & Mesangial cells \\
\hline 127 & DUOX2 & 64 & Dual oxidase 2 & Thyroid \\
\hline 3 & GDPD2 & 68 & Glycerophosphodiester phosphodiesterase containing 2 & Osteoblasts \\
\hline 3 & PDEIIA & 75 & Phosphodiesterase IIA & Testis \\
\hline 2 & CLDN23 & 76 & Claudin 23 & Placenta \\
\hline I & PLCL4 & 78 & Phospholipase C-like 4 & Neurons \\
\hline 1 & EYA4 & 81 & Eyes absent homolog 4 & Heart and cochlea \\
\hline I & LIPH & 85 & Lipase, member $\mathrm{H}$ & Intestine \\
\hline 1 & $R B P 3$ & 88 & Retinol binding protein 3 & Retina \\
\hline
\end{tabular}

epidermis had not been previously assessed, evoking the possibility that they might play a specific role in this tissue.

\section{Real-time PCR expression profiling of selected genes}

Genes involved in the establishment of the skin barrier are expected to be specifically overexpressed by granular keratinocytes. To compare the expression levels of candidate genes between the basal layer and GKs, quantitative real-time PCR experiments were performed with the $\mathrm{T}_{4}$ and $\mathrm{T} 1$ cell fractions. Based on predicted domains and homologies, 73 genes represented by less than 100 ESTs were selected (Table 7). The relative $\mathrm{T} 4 / \mathrm{T} 1$ ratio could not be calculated for 20 of them due to very low expression levels. Ten genes were equally expressed in the two layers, and nine were overexpressed in the basal layer, even if expressed at a low level in the granular layer. Interestingly, 33 were overexpressed in the granular layer with T4/T1 ratios ranging from 6 to 800 . For several genes, the $\mathrm{T} 4 / \mathrm{T} 1$ expression ratio was thus much larger than that observed for the $K L K 7$ gene, used as a specific marker of the GKs in our cell purification experiments (Table
1). Therefore, these data emphasize the high degree of purity of the GKs we have purified from healthy human skin. They also provide one with new, highly specific markers for this cell type.

\section{Identification of new genes \\ FLG2}

The epidermal differentiation complex (EDC) spans 1.62 megabases on 1q21.3 and contains approximately 50 genes specifically involved in the barrier function, such as those encoding involucrin, loricrin, filaggrin, small proline rich proteins (SPRR1-4) or late cornified envelope proteins (LCE1-5) (Figure 3a). We cloned many sequences corresponding to known genes of this locus (Figure $3 \mathrm{~b}$ ), but also a large number of sequences for a previously poorly characterized transcript encoding filaggrin 2 (FLG2; also called ifapsoriasin (IFPS); (GenBank:AY827490)). FLG2 displays features of the fused-family genes (encoding filaggrin, trichohyalin, or repetin), with three exons and a large predicted protein sequence $(2,391$ amino acids) containing two 
Table 5

\begin{tabular}{|c|c|c|c|}
\hline No. of ORESTES & Gene symbol & No. of UniGene ESTs & Full name \\
\hline 13 & ASAH3 & 4 & $\mathrm{~N}$-acylsphingosine amidohydrolase 3 \\
\hline I & LIPL2 (LIPK) & 4 & Lipase-like, ab-hydrolase domain containing 2 \\
\hline 11 & CLEC2A & 7 & C-type lectin domain family 2 , member $A$ \\
\hline 2 & IGFL3 & 12 & Insulin growth factor-like family member 3 \\
\hline 3 & LYG2 & 14 & Lysozyme-like \\
\hline I & PNPLAI & 15 & Patatin-like phospholipase domain containing I \\
\hline 9 & GSDM I & 16 & Gasdermin I \\
\hline I & GRID2IP & 17 & Glutamate receptor, ionotropic, delta 2 interacting protein \\
\hline 2 & ILIF7 & 17 & Interleukin I family, member 7 \\
\hline 2 & FCRL6 & 17 & Fc receptor-like 6 \\
\hline 3 & AADACL2 & 20 & Arylacetamide deacetylase-like 2 \\
\hline I & LIPL3 (LIPM) & 20 & Lipase-like, ab-hydrolase domain containing 3 \\
\hline 1 & LAMB4 & 26 & Laminin, beta 4 \\
\hline 10 & THEM5 & 26 & Thioesterase superfamily member 5 \\
\hline 1 & FLJ 90165 & 27 & Gamma-glutamyltransferase 6 homolog \\
\hline 3 & FLJ4565I & 28 & Phospholipase A2, group IVE \\
\hline 1 & LPIN3 & 31 & Lipin 3 \\
\hline I & SLC25A34 & 36 & Solute carrier family 25 , member 34 \\
\hline I & GPRII5 & 37 & G protein-coupled receptor 115 \\
\hline 1 & LRP5L & 41 & Low density lipoprotein receptor-related protein 5-like \\
\hline I & HSPCI05 & 45 & NAD $(P)$ dependent steroid dehydrogenase-like \\
\hline I & QPCTL & 52 & Glutaminyl-peptide cyclotransferase-like \\
\hline 3 & PLA2G4F & 56 & Phospholipase A2, group IVF \\
\hline I & KIAA0605 & 58 & ADAMTS-like 2 \\
\hline I & CTGLFI & 60 & Centaurin, gamma-like family, member I \\
\hline 2 & UGT3A2 & 65 & UDP glycosyltransferase 3 family, polypeptide A2 \\
\hline 1 & GALNTI 7 & 74 & Polypeptide $\mathrm{N}$-acetylgalactosaminyltransferase 17 \\
\hline 1 & BAIAP2L2 & 76 & BAll-associated protein 2-like 2 \\
\hline I & FLJ43692 & 80 & ARHGEF5-like \\
\hline 1 & VILL & 86 & Villin-like \\
\hline I & LOC203427 & 87 & Similar to solute carrier family 25, member 16 \\
\hline 1 & ILI TRE & 100 & Interleukin 17 receptor $\mathrm{E}$ \\
\hline
\end{tabular}

calcium binding EF-hand domains and a large domain made of repeated segments of about 25 amino acids. The amino acid composition of FLG2 is very similar to that of filaggrin, with a high content of serine (22\%), glycine (20\%), histidine (10\%) and glutamine (10\%). The expression of this gene is likely restricted to the epidermis, as shown by PCR on a panel of cDNAs from 16 healthy human tissues and organs (Figure 4). Real-time PCR also showed a strong overexpression of the FLG2 gene in GKs, with a T4/T1 ratio of 800 (Table 7). These results thus suggest that this gene is a new functional member of the EDC complex, in agreement with its similarity to the filaggrin gene, whose function in the epidermal barrier is well established.
Lipase-like genes

Two ORESTES were identified as the human orthologues of the murine lipases Lipl2 (NM_172837) and Lipl3 (BCo31933), previously identified by large-scale mouse cDNA sequencing by the Riken Institute [33] and the Mammalian Gene Collection program [34], respectively. The corresponding human genes LIPL2 and LIPL3 were clustered in a $665 \mathrm{kB}$ interval on chromosome $10 \mathrm{q} 23.31$ with genes encoding two experimentally characterized lipases, LIPA (lysosomal acid lipase, MIM +27,800o) and LIPF (gastric lipase, MIM \#601980) and two hypothetical lipase-like proteins, LIPL1 and LIPL4 (Figure 5a). Therefore, our study contributed to the elucidation of a specialized human genomic locus that includes six lipase genes and four other genes (ANKRD22, STAMBPL1, ACT2 and FAS) of apparently unrelated function (Figure 5a). In accordance with the Hugo Gene 
Table 6

Unknown genes with 100 or less UniGene ESTs

\begin{tabular}{|c|c|c|c|}
\hline No. of ORESTES & Gene symbol & No. of UniGene ESTs & Full name \\
\hline 1 & FLJ4386I & 3 & Flj4386I \\
\hline 1 & LOC38979I & 3 & Hypothetical gene supported by AK094537 \\
\hline 1 & LOC285435 & 4 & Hypothetical LOC285435 \\
\hline 2 & LOC387846 & 6 & Hypothetical LOC387846 \\
\hline 4 & LOC401062 & 6 & Hypothetical gene supported by AK092973 \\
\hline I & IMAGE:52609/4 & 7 & Image:52609|4 \\
\hline 5 & LOC338667 & 7 & Hypothetical protein LOC338667 \\
\hline 5 & PSORSIC2 & 8 & Psoriasis susceptibility I candidate 2 \\
\hline 1 & DKFZp779B 1540 & 9 & Hypothetical protein dkfzp779b|540 \\
\hline 5 & Cl4orf72 & 9 & Chromosome 14 open reading frame 72 \\
\hline 1 & FLJ37989 & 10 & Flj37989 \\
\hline 56 & FLG2 & 10 & Filaggrin 2 (ifapsoriasin) \\
\hline 10 & WFDC5 & 11 & WAP four-disulfide core domain 5 \\
\hline 1 & LOC402IIO & 12 & Hypothetical LOC402110 \\
\hline 1 & PLEKHNI & 12 & Pleckstrin homology domain containing, family $\mathrm{N}$ member I \\
\hline I & LOC44 I 240 & 13 & Hypothetical protein LOC44I 240 \\
\hline 4 & FLJ38159 & 14 & Hypothetical protein FLJ38I59 \\
\hline 1 & ClorfI 77 & 15 & Chromosome I open reading frame 177 \\
\hline 1 & HMCN2 & 16 & Hemicentin 2 \\
\hline 2 & MGC23985 & 16 & Similar to AVLV472 \\
\hline 1 & OFCCI & 17 & Orofacial cleft I candidate I \\
\hline 1 & LOC44I860 & 17 & Novel KRAB box containing $\mathrm{C} 2 \mathrm{H} 2$ type zinc finger protein \\
\hline 5 & AMIGO3 & 18 & Adhesion molecule with Ig-like domain 3 \\
\hline 1 & LOC44I 257 & 20 & Hypothetical protein LOC44I 257 \\
\hline I & LOC285484 & 20 & Hypothetical protein LOC285484 \\
\hline 1 & C20orf9l & 20 & Chromosome 20 open reading frame 91 \\
\hline 1 & LOC202460 & 21 & Hypothetical protein LOC202460 \\
\hline 2 & FLJ25664 & 21 & Flj25664 \\
\hline 8 & FLJ4 I 623 & 21 & Flj4 I 623 \\
\hline 10 & LOC342897 & 21 & Similar to F-box only protein 2 \\
\hline 1 & LOC339237 & 23 & Similar to Envoplakin \\
\hline 13 & LOCI 26248 & 24 & Hypothetical protein LOCI26248 \\
\hline 1 & LOC389/42 & 27 & Hypothetical LOC389142 \\
\hline 3 & C20orf95 & 28 & Chromosome 20 open reading frame 95 \\
\hline I & FKBP9L & 31 & FK506 binding protein 9-like \\
\hline I & FNDC8 & 31 & Fibronectin type III domain containing 8 \\
\hline 3 & FLJ463II & 31 & FLJ463II protein \\
\hline 1 & C3orf47 & 33 & Chromosome 3 open reading frame 47 \\
\hline 1 & LOC283/43 & 35 & Hypothetical protein LOC283I43 \\
\hline 1 & LOC388727 & 35 & Hypothetical LOC388727 \\
\hline 2 & FLJ44317 & 35 & Flj443 I 7 \\
\hline 1 & $F L J 31 / 84$ & 36 & Flj31I84 \\
\hline I & $\mathrm{LOCI} 25893$ & 39 & Hypothetical protein LOCI 25893 \\
\hline 1 & ZNF3II & 40 & Zinc finger protein 311 \\
\hline 1 & BC04/923 & 40 & Image:5300|99 \\
\hline 2 & ZNF600 & 43 & Zinc finger protein 600 \\
\hline 3 & $\mathrm{MCMDCl}$ & 43 & Minichromosome maintenance deficient domain containing I \\
\hline 3 & FLJ 13646 & 46 & Hypothetical protein FLJI3646 \\
\hline 1 & $\mathrm{Cl} 4 \mathrm{orfl} 2 \mathrm{I}$ & 48 & Chromosome 14 open reading frame 121 \\
\hline I & FAM83F & 49 & Family with sequence similarity 83, member $F$ \\
\hline 3 & ABHD9 & 51 & Abhydrolase domain containing 9 \\
\hline 1 & LOCI34466 & 52 & Hypothetical protein LOCI34466 \\
\hline I & CXorf33 & 52 & Chromosome $X$ open reading frame 33 \\
\hline
\end{tabular}


Table 6 (Continued)

\section{Unknown genes with 100 or less UniGene ESTs}

\begin{tabular}{|c|c|c|c|}
\hline 2 & FLJ25006 & 52 & Hypothetical protein FLJ25006 \\
\hline 2 & DKFZp434N062 & 53 & Hypothetical protein dkfzp434n062 \\
\hline 9 & LASS3 & 53 & LAGI longevity assurance homolog 3 \\
\hline 1 & Cl4orf2I & 54 & Chromosome 14 open reading frame 21 \\
\hline 1 & $\mathrm{Cl} 7$ orf67 & 56 & Chromosome 17 open reading frame 67 \\
\hline 1 & FAM62C & 58 & Family with sequence similarity 62 , member $C$ \\
\hline 2 & $\mathrm{Cl} 4$ orf29 & 60 & Chromosome 14 open reading frame 29 \\
\hline 1 & FLJ21736 & 61 & Esterase 31 \\
\hline 3 & LOC349II4 & 61 & Hypothetical protein LOC349II4 \\
\hline 1 & MGC26885 & 62 & Hypothetical protein MGC26885 \\
\hline 1 & SMA3 & 62 & Sma3 \\
\hline 8 & FAM $46 B$ & 62 & Family with sequence similarity 46 , member $B$ \\
\hline 13 & ELMODI & 62 & ELMO/CED-12 domain containing I \\
\hline 3 & DENND2C & 63 & DENN/MADD domain containing $2 \mathrm{C}$ \\
\hline 13 & ANKRD35 & 64 & Ankyrin repeat domain 35 \\
\hline 5 & LOC401553 & 66 & Hypothetical gene supported by $\mathrm{BC} 019073$ \\
\hline 1 & LOC390927 & 67 & Similar to zinc finger protein 569 \\
\hline 1 & ZNF696 & 67 & Zinc finger protein 696 \\
\hline 2 & CCDC9 & 69 & Coiled-coil domain containing 9 \\
\hline 6 & Cl5orf40 & 70 & Chromosome 15 open reading frame 40 \\
\hline 1 & $L O C I 48137$ & 73 & Hypothetical protein BC0I7947 \\
\hline 1 & $\mathrm{ZC} 3 \mathrm{H} / 2 \mathrm{C}$ & 74 & Zinc finger $\mathrm{CCCH}$-type containing $12 \mathrm{C}$ \\
\hline 1 & $A P X L 2$ & 74 & Apical protein 2 \\
\hline 1 & ZMYNDI9 & 75 & Zinc finger, MYND-type containing 19 \\
\hline 1 & LRRC37B & 77 & Leucine rich repeat containing 37B \\
\hline 2 & FLJ32356 & 77 & Family with sequence similarity 109 , member $\mathrm{A}$ \\
\hline 3 & $D Q X I$ & 77 & DEAQ box polypeptide I \\
\hline 2 & C9orf9 & 79 & Chromosome 9 open reading frame 9 \\
\hline 4 & FNDC6 & 79 & Fibronectin type III domain containing 6 \\
\hline 1 & MSTP9 & 82 & Macrophage stimulating, pseudogene 9 \\
\hline 1 & HES2 & 83 & Hairy and enhancer of split 2 \\
\hline 1 & FLJ37464 & 84 & Hypothetical protein FLJ37464 \\
\hline 1 & KIAAI862 & 84 & KIAAI 862 protein \\
\hline 1 & LOC196264 & 86 & Hypothetical protein LOCI96264 \\
\hline 1 & Clorf5I & 86 & Chromosome I open reading frame $5 \mathrm{I}$ \\
\hline 2 & ANKRD5 & 86 & Ankyrin repeat domain 5 \\
\hline 1 & CXorf23 & 87 & Chromosome $X$ open reading frame 23 \\
\hline 2 & SMCR8 & 88 & Smith-Magenis syndrome chromosome region, candidate 8 \\
\hline 2 & DKFZp686LI8I4 & 88 & Hypothetical protein dkfzp68611814 \\
\hline 2 & MGC34647 & 88 & Hypothetical protein MGC34647 \\
\hline 1 & C6orfl05 & 89 & Chromosome 6 open reading frame 105 \\
\hline 1 & FLJ23186 & 90 & Chromosome 3 open reading frame 52 \\
\hline 1 & SAMDIO & 91 & Sterile alpha motif domain containing 10 \\
\hline 2 & KIAA/287 & 91 & Kiaa 1287 \\
\hline 2 & Cl9orf36 & 91 & Chromosome 19 open reading frame 36 \\
\hline 1 & ZNF662 & 93 & Zinc finger protein 662 \\
\hline 2 & ZNF429 & 97 & Zinc finger protein 429 \\
\hline 12 & SMPD3 & 98 & Sphingomyelin phosphodiesterase 3 \\
\hline 1 & PCGFI & 100 & Polycomb group ring finger I \\
\hline 1 & CI7orf6I & 100 & Chromosome 17 open reading frame 61 \\
\hline 1 & KIAAI853 & 100 & Kiaal853 \\
\hline 1 & C22orf23 & 100 & Chromosome 22 open reading frame 23 \\
\hline
\end{tabular}


Table 7

\section{Comparison of gene expression in the T4 and TI cell fractions by real-time PCR}

\begin{tabular}{|c|c|c|c|c|}
\hline No. of ORESTES & Gene symbol & Full name & No. of UniGene ESTs & $\mathrm{T} 4 / \mathrm{TI}$ expression ratio \\
\hline 56 & FLG2 & Filaggrin 2 (ifapsoriasin) & 10 & 800 \\
\hline 9 & GSDMI & Gasdermin I & 16 & 800 \\
\hline 13 & ASAH3 & $\mathrm{N}$-acylsphingosine amidohydrolase 3 & 4 & 300 \\
\hline 2 & ILIF7 & Interleukin I family, member 7 & 17 & 200 \\
\hline 13 & ELMODI & ELMO/CED- 12 domain containing I & 62 & 160 \\
\hline 0 & LIPL4 (LIPN) & Lipase-like, ab-hydrolase domain containing 4 & 9 & 150 \\
\hline 1 & LIPL3 (LIPM) & Lipase-like, ab-hydrolase domain containing 3 & 20 & 130 \\
\hline 3 & C20orf95 & Chromosome 20 open reading frame 95 & 28 & 120 \\
\hline 1 & WFDCI 2 & WAP four-disulfide core domain 12 (WAP2) & 3 & 110 \\
\hline 1 & LIPL2 (LIPK) & Lipase-like, ab-hydrolase domain containing 2 & 4 & 100 \\
\hline 12 & SMPD3 & Sphingomyelin phosphodiesterase 3 & 98 & 70 \\
\hline 10 & LOC440449 & Similar to WDNMI homolog (LOC645638) & - & 50 \\
\hline 99 & SERPINAI2 & Serpin peptidase inhibitor, clade A, member 12 & 11 & 50 \\
\hline I & PII & 26 serine protease & 64 & 35 \\
\hline 5 & PSORSIC2 & Psoriasis susceptibility I candidate 2 & 8 & 30 \\
\hline 1 & C3orf52 & Chromosome 3 open reading frame 52 & 90 & 30 \\
\hline 2 & CLDN23 & Claudin 23 & 76 & 25 \\
\hline 1 & PNPLAI & Patatin-like phospholipase domain containing I & 15 & 20 \\
\hline 10 & THEM5 & Thioesterase superfamily member 5 & 26 & 20 \\
\hline 3 & $A B H D 9$ & Abhydrolase domain containing 9 & 51 & 20 \\
\hline 2 & TMEM /6H & Transmembrane protein $16 \mathrm{H}$ & 78 & 16 \\
\hline 6 & SERPINBI2 & Serpin peptidase inhibitor, clade B, member 12 & 6 & 15 \\
\hline 1 & PLEKHNI & Pleckstrin homology domain containing, family $\mathrm{N}$ member I & 12 & 12 \\
\hline 1 & FAM83F & Family with sequence similarity 83 , member $F$ & 49 & 12 \\
\hline 3 & AADACL2 & Arylacetamide deacetylase-like 2 & 20 & 12 \\
\hline 10 & LOC342897 & Similar to F-box only protein 2 & 21 & 10 \\
\hline 9 & LASS3 & LAGI longevity assurance homolog 3 & 53 & 10 \\
\hline 20 & CASZI & Castor homolog I, zinc finger & 61 & 10 \\
\hline 8 & FAM46B & Family with sequence similarity 46 , member $B$ & 62 & 10 \\
\hline 14 & SERPINB7 & Serpin peptidase inhibitor, clade B, member 7 & 64 & 10 \\
\hline 1 & CARD $/ 4$ & Caspase recruitment domain family, member 14 & 86 & 8 \\
\hline 1 & GGT6 & Gamma-glutamyltransferase 6 homolog & 27 & 6 \\
\hline I & CXorf33 & Chromosome $X$ open reading frame 33 & 52 & 6 \\
\hline 10 & WFDC5 & WAP four-disulfide core domain 5 (WAPI) & 11 & I \\
\hline 5 & KIAA05 I 4 & Kiaa05।4 & 16 & 1 \\
\hline 5 & AMIGO3 & Adhesion molecule with Ig-like domain 3 & 18 & I \\
\hline 3 & PLA2G4E & Phospholipase A2, group IVE & 28 & 1 \\
\hline 1 & HSPCIO5 & NAD(P) dependent steroid dehydrogenase-like & 45 & 1 \\
\hline 3 & PLA2G4F & Phospholipase A2, group IVF & 56 & 1 \\
\hline 127 & DUOX2 & Dual oxidase 2 & 64 & 1 \\
\hline 1 & $A P X L 2$ & Apical protein 2 & 74 & 1 \\
\hline 3 & RAB38 & RAB38, member RAS oncogene family & 79 & 1 \\
\hline 1 & AFMID & Arylformamidase & 87 & 1 \\
\hline 2 & ANKRD5 & Ankyrin repeat domain 5 & 86 & 0.46 \\
\hline 3 & $\mathrm{MCMDCl}$ & Minichromosome maintenance deficient domain containing I & 43 & 0.4 \\
\hline 1 & $\mathrm{Cl} 4$ orf2I & Chromosome 14 open reading frame 21 & 54 & 0.38 \\
\hline 3 & LEAP-2 & Liver-expressed antimicrobial peptide 2 & 53 & 0.36 \\
\hline 1 & $L R P 5 L$ & Low density lipoprotein receptor-related protein 5-like & 41 & 0.35 \\
\hline 4 & FNDC6 & Fibronectin type III domain containing 6 & 79 & 0.3 \\
\hline 2 & KIAA $/ 287$ & Kiaa I 287 & 91 & 0.28 \\
\hline
\end{tabular}

Not determined: FLJ2 I 736, HES2, ZNF696, LYG2, MGC34829, KIAA0963, ALKBH, ADAMTSL2, c6orfI 05, AW367250, KCNJI2, LOC338667, TMPRSS5, CLEC2A, FLJ2 I736, cl 4orf29, cl 4orf72, KRBAI, FLJ37464, LOCI34466. 


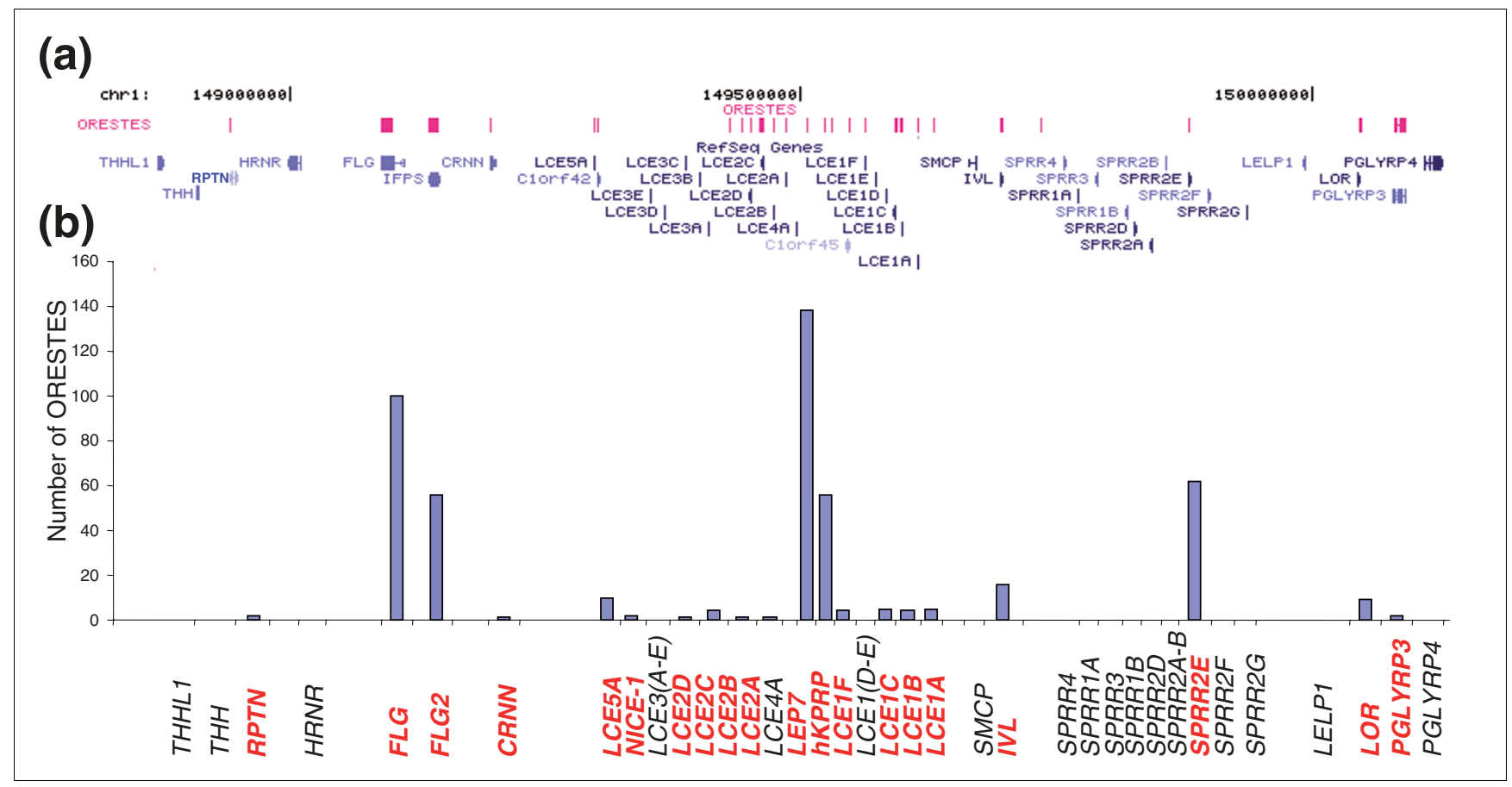

Figure 3

Genes of the EDC present in the ORESTES dataset. (a) Screen copy of a UCSC Genome Browser window (chrl:I50,300,000-15I,590,000; hg I7, May 2004) showing the RefSeq genes from the EDC, and the ORESTE custom track. (b) Number of ORESTES for each gene of the locus. The genes for which at least one ORESTE was sequenced are in red bold characters.

Nomenclature Committee (HGNC), these new hypothetical lipase genes, LIPL1, LIPL2, LIPL3 and LIPL4, have been renamed $L I P J, L I P K, L I P M$, and $L I P N$, respectively.

Rapid amplification of cDNA ends (RACE) cloning experiments of the human LIPK mRNA localized the cap site $165 \mathrm{nt}$ upstream of the conceptual ATG initiation codon. For the LIPM and LIPN mRNAs, the entire coding sequences have been cloned using primers deduced from alignments with the mouse cDNAs (GenBank:EF426482, GenBank:EF426484 and GenBank: EF426483).

The conceptual translation of LIPJ, LIPK, LIPM, and LIPN ORFs led to proteins of $366,399,423$ and 398 amino acids, respectively. As is the case for LIPA and LIPF, these proteins contain an abhydrolase associated lipase region (PFAM PFo4083) with an alpha/beta hydrolase fold domain (PFAM PFo0561). More precisely, they display the characteristic SerAsp-His catalytic triad (Figure 5b) also present in various hydrolases, such as serine proteases [35,36]. Surprisingly, LIPJ (NM_o01010939) appeared as the only member in this family to lack a cleavable signal peptide (Figure $5 \mathrm{~b}$ ) and might thus play a distinct role in human physiology.

The clustering of six lipase genes on the human 10q23.31 locus led us to examine in detail the syntenic mouse locus (19qC1), where the orthologous genes except for $L I P N$ and $L I P J$ had already been identified or predicted,. Sequence analysis showed that the AK154333 cDNA clearly corresponds to the LIPN orthologue ( $89 \%$ homology). As in the human genome, this gene resides between the Lipl2 and Lipl3 genes. Intriguingly, we could not precisely identify a single orthologue of LIPJ. Instead, we found upstream of the Lipf gene a cluster of five predicted genes encoding secreted alpha/beta hydrolases as well as at least three pseudogenes containing premature stop codons (Figure 5a). A syntenic cluster, albeit of smaller size, resides in the rat genome. These five hypothetical functional mouse lipases form by themselves a separate group in the phylogenic tree (Figure $5 \mathrm{c}$; Additional data file 4). Their genomic localization suggests that they could have arisen from a large expansion by tandem duplications well after the separation of the rodents from the primate ancestors, or from a lipase gene lost in the human genome. These five new genes have thus been renamed Lipdc1-5 for lipase domain containing1-5 in accordance with the Mouse Gene Nomenclature Committee (MGNC) (Additional data file 5).

The human LIPK, LIPM and LIPN genes appear to be exclusively expressed in the epidermis, as shown by PCR on a panel of $16 \mathrm{cDNAs}$ (Figure 4). Their expression was highly specific for GKs, with real-time PCR T4/T1 ratios of 100-150 (Table 7). LIPJ expression was also detected in the epidermis by PCR, but was too weak to allow real-time PCR experiments to be performed. These results strongly suggest that the LIPK, $L I P M$ and $L I P N$ genes play a highly specific role in the last 


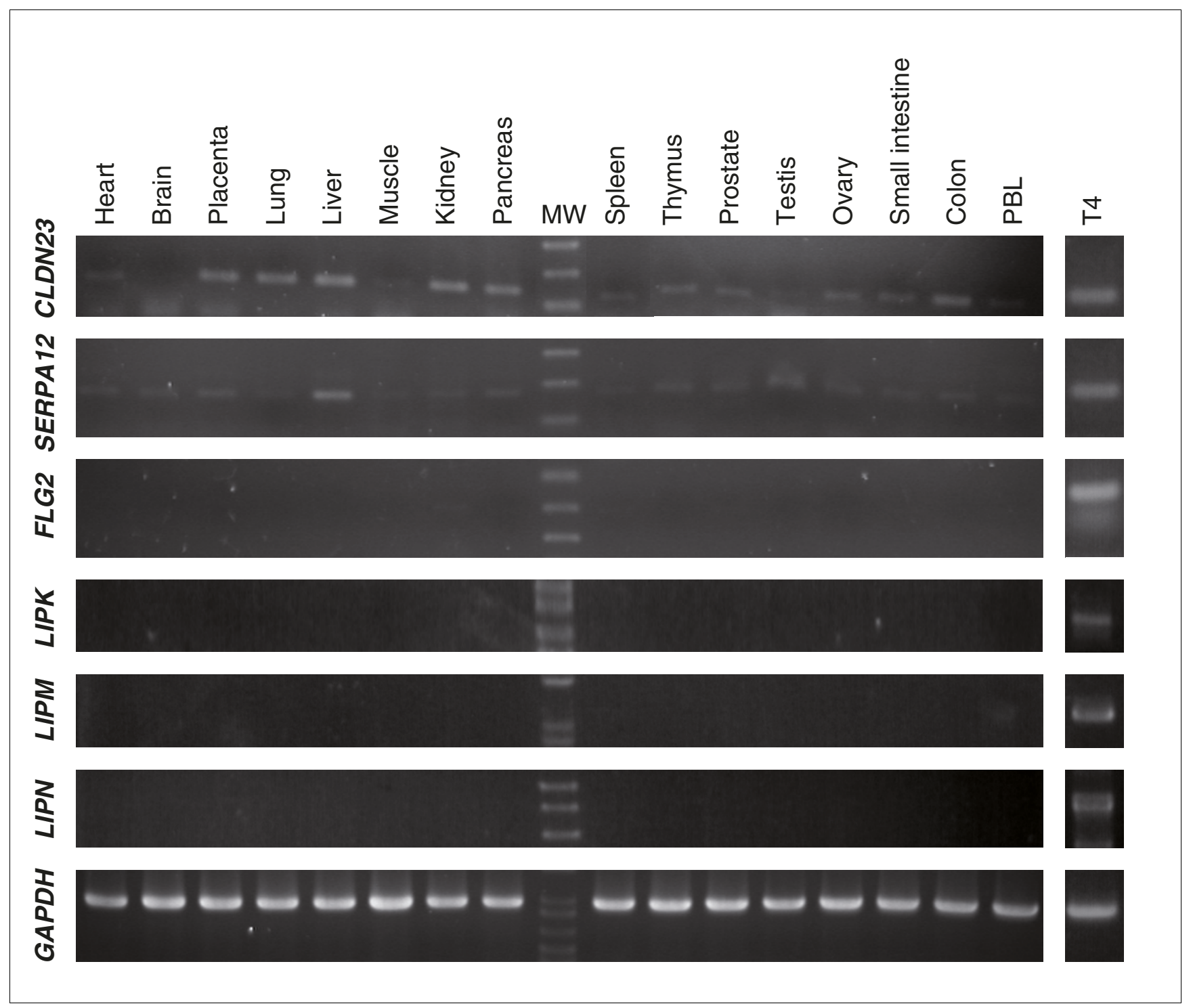

Figure 4

Expression profile of newly identified genes. PCR experiments were performed with a commercial panel of cDNAs from 16 human tissues (PBL, peripheral blood leukocytes) and with cDNAs prepared from the T4 fraction enriched in GKs. For each gene, PCR primers were chosen to amplify a cDNA fragment encompassing at least two exons. Note the highly specific expression pattern of FLG2, LIPK, $M, N$, and, to a lesser extent, SERPINAI 2 genes. The apparent size variation of the CLDN23 fragment results from an artifactual gel distortion. Expression of GAPDH, assessed with the primers provided by the manufacturer, was used as a control.

step of keratinocyte differentiation. Although highly related, the LIPJ protein might play a different role, as previously suggested by its lack of a signal peptide.

\section{Clorf8I}

Six spliced ORESTES corresponded to a totally unknown gene without mRNA sequences in the databases. We performed RACE experiments and identified 16 exons and 15 introns with canonical splice sites and a consensus polyadenylation signal (GenBank:DQ983818). In agreement with the HGNC, we named this gene C1orf81. To date, only 16 ESTs are present in the databases, one from testis and the others from pooled tissues (Additional data file 1). PCR on a panel of cDNAs from 16 healthy human tissues and organs showed that spliced transcripts for this gene are detectable in most samples (Figure 6a). From its conceptual translation, the C1orf81 mRNA might encode a 373 amino acid protein. However, a second ORF was present in another frame that overlapped the first one by 87 amino acids. This suggested that a longer protein might be produced by a ribosomal frameshift mechanism. We explored the C1orf81 gene orthologues in vertebrate genomes, and particularly the region corresponding to the ORF overlap (exons 7-9). Compared with the C1orf 81 gene from 10 other mammals, exon 8 of the 
human gene contained a 1 base-pair insertion that creates the frameshift (Figure 6b). The possibility of a sequence error in the present human genome assembly was excluded by sequencing the exon 8 region from two individuals. Accordingly, the human C1orf 81 gene product would be 373 amino acids long, but 762 and 714 amino acids long in the chimpanzee and rhesus monkey, respectively. In all cases, the analysis of the peptide sequence did not reveal known domains or signal peptide. Further studies are needed to uncover the role of this new gene in mammalian physiology, and evaluate the consequences of its possible inactivation in human.

\section{Discussion}

We have described here the first large-scale study of the transcriptome of human epidermal cells. As we are interested in genes that participate in the barrier function of the skin, we focused on GKs. They correspond to the ultimate step in the course of keratinocyte differentiation, and are the last epidermal cells to display gene expression activity before undergoing a particular programmed cell death leading to cornification. Because these cells represent less than $10 \%$ of the epidermis population, a preliminary step was to design an efficient purification method starting from healthy human skin fragments. After unsuccessful attempts using size filtration, Ficoll gradients, and fluorescence-activated cell sorting, we used successive short-term enzyme incubations to progressively detach cells from the deep layers, and purify the cells that remain attached to the cornified layer. These incubations were performed at $4^{\circ} \mathrm{C}$ to stop cellular metabolic activity and preserve the mRNA pool from degradation. This point is highly relevant as many growth factors, cell cycle regulators and transcription factors are encoded by short-lived mRNA. Quantitative PCR experiments were used to assess the relative expression levels of several genes in the successive cell fractions.

\section{ORESTE technique}

In the present transcriptome project, the ORESTE methodology was selected because it produces sequences that are distributed predominantly within the central part of the corresponding transcripts and is biased towards less-abundant mRNAs [9]. When using arbitrarily chosen primers for reverse transcription and $\mathrm{PCR}$, the amplification of a given transcript is proportional to its length and to the probability for the primer to anneal at low stringency $\left(37^{\circ} \mathrm{C}\right)$. This conceptual normalization was secondarily strengthened by removing cDNA libraries with highly abundant amplification products. After the elimination of irrelevant sequences, we produced 16,591 ORESTES representing 3,387 genes. The distribution of the ORESTE number per gene (Figure 2) and the low number of ESTs in the corresponding UniGene entries fully confirmed that the transcript normalization obtained with this method is at least comparable to that of hybridization-subtraction techniques (for an example, see [37]).

\section{Overrepresented genes}

Nevertheless, a few genes were represented by more than 100 sequences obtained from different mini-libraries. These include ubiquitous, highly expressed genes like RPS8 and $E E F 1 A 1$, and genes already known to be highly transcribed in GKs (those encoding keratin 1, late envelope protein 7 or profilaggrin). Other members of this class, $D M K N$ (formerly ZD52F1O), SERPINA12 and FLG2 were not previously known to be overexpressed in the skin, and were thus studied in more detail. Two isoforms of transcripts for the dermokine gene were previously reported $[15,16]$ and we describe 13 novel mRNA isoforms that are either ubiquitous or epidermis-specific depending upon alternative promoter usage. The epidermis-specific forms encode secreted proteins of still unclear function, and are abundantly transcribed in this tissue [17].

SERPINA12, also named Vaspin (visceral adipose tissuederived serine protease inhibitor), is an extracellular serine protease inhibitor that displays insulin-sensitizing effects [38]. In addition to the previously documented expression in the liver, we observed that the SERPINA12 gene is highly expressed in the human epidermis (Figure 4). Moreover, realtime PCR showed that it is specifically expressed by GKs (T4/ T1 ratio of 50). The Serpin A12 protein might thus play a role in the regulation of the complex balance between various proteases and their inhibitors operative in the desquamation process.

The filaggrin 2 (FLG2; or ifapsoriasin (IFPS)) gene is a new member of the EDC (chromosome 1q21.3), a cluster of approximately 50 genes involved in cornification. Our realtime RT-PCR experiments revealed that FLG2 expression displays strong specificity for GK. Interestingly, its composition is very close to that of filaggrin. The degradation of filaggrin is considered to be at the origin of the free amino acid pool of the natural moisturizing factor, which is capable of attracting and retaining water in the cornified layer to achieve skin softness and flexibility. We suggest that FLG2 might have a similar function.

\section{Clorf8 I might have lost its function during hominization}

A new gene characterized in this study, C1orf81, is particularly intriguing as the corresponding transcript (2,284 nt) displays an ORF disruption by a human-specific, single nucleotide insertion in the eighth exon (Figure 6b). As a result, a premature stop codon resides in exon 9, 103 nt upstream of its 3' end. Consequently, the human C1orf81 mRNA might be degraded by the nonsense mediated mRNA decay (NMD) pathway [39]. This appears unlikely, however, as its expression in various tissues was detected by RT-PCR (Figure 6a). However, its expression in epidermis was too weak to 
perform real-time PCR experiments. Our analysis suggests that the human C1orf81 gene encodes a truncated protein relative to other mammalian species, including the rhesus monkey. Nevertheless, we do not exclude the possibility that the translation of the human C1orf81 mRNA might produce a full-length protein by a +1 ribosomal frameshift mechanism. To our knowledge, such a phenomenon has been uniquely described in human for the ornithine decarboxylase antizyme gene family [40]. This would thus constitute a further example of a (partial) loss of gene function during hominoid evolution. The systematic comparison of the human and chimpanzee genomes has revealed nine other human-specific frameshift mutations (pseudogenization) leading to the mRNA decay or carboxy-terminal protein truncation [41]. Gene losses that occurred after human-chimpanzee divergence could play a role in adaptive evolution, as shown for caspase 12 inactivation during hominization [42]. In this framework, it is intriguing that a member of the hair keratin gene complex, KRTHAP1, and a serine protease inhibitor gene, SERPINA13, are also among the few genes specifically inactivated in human by a mutation $[43,44]$.

In addition, we sequenced ORESTES for CTAGE5 and $C N O T 6 L$ functional retrogenes, which are specific for primate and hominoid lineages, respectively. Moreover, the CTAGE5 retrogene is included in a larger, human-specific insertion (data not shown). It is indeed known that new genes have emerged after a burst of retroposition in primates [45]. Not surprisingly, this suggests that modifications of genes expressed in the skin participate in obvious differences between human and chimpanzee.

\section{Real-time PCR experiments}

Real-time PCR experiments were used to measure the relative expression ratio of selected genes in the $\mathrm{T}_{4}$ (mainly GKs) and T1 (basal layer) fractions. In initial experiments using the $S C C E / K L K 7$ gene as a GK specific marker, the $\mathrm{T}_{4} / \mathrm{T} 1$ ratios (54-189; Table 1) indicated that the $\mathrm{T}_{4}$ fraction was indeed highly enriched in GKs. However, subsequent experiments led to even higher ratios (for example, 80o for FLG2 and $G S D M$ ). Our approach thus led to the discovery of new, exquisitely specific gene markers for GKs that constitute valuable tools for detailed studies of epidermis architecture by histochemistry and in situ hybridization methods. Moreover, such highly specific expression strongly suggests that the corresponding genes play key roles in barrier function. The real- time PCR studies also revealed equal expression of several genes in the $\mathrm{T}_{4}$ and $\mathrm{T} 1$ fractions, suggesting that they are transcribed during all steps of keratinocyte differentiation. In addition to providing one with a highly purified GK fraction, our human skin fractionation method thus constitutes a new tool for the characterization of genes involved in the successive steps of terminal differentiation in the epidermis.

\section{GK-specific candidate genes}

To further characterize genes poorly represented in databases, the 330 genes with the lowest EST number in the UniGene database ( $\leq 100$ ESTs) were analyzed in more detail. Among these, the known specific genes involved in keratinocyte terminal differentiation account for only $15 \%$ of the panel, whereas $42 \%$ (139) encode hypothetical proteins. This shows that genes expressed specifically in the uppermost layers of the epidermis are poorly represented in the sequence databases, and suggests that some genes encoding hypothetical proteins may play a functional role in the late steps of epidermal differentiation. We specially focused on genes potentially involved in desquamation regulation as well as lipid metabolism and transport, considering their importance for barrier function establishment. Thus, 73 candidate genes were chosen for a quantitative study of their expression in the course of epidermal differentiation by real-time RT-PCR. The $\mathrm{T} 4 / \mathrm{T} 1$ ratio for 20 of them could not be calculated by quantitative RT-PCR using the SYBR Green method, due to a very low expression level. This underlines the sensitivity of the ORESTES method in detecting rare transcripts. Among the 52 remaining genes, real-time RT-PCR experiments revealed that 33 are upregulated during late epidermis differentiation, and hypotheses on the function of some of them are presented below. Moreover, the expression pattern of 6 of these genes is mostly restricted to epidermis as shown by PCR on a panel of 16 human cDNAs prepared from various organs (Figure 4). Among the 330 genes poorly represented in EST databases that we identified, a significant proportion of granular keratinocyte specific genes are thus suspected to be present.

\section{Expression of proteases and protease inhibitors in the human epidermis}

The balance between proteases and protease inhibitors is essential to desquamation [46]. The inactivation of the protease inhibitor cystatin M/E gene in mice causes lethality and defects in epidermal cornification [47]. Mutations in the SPINK5 gene (encoding another protease inhibitor) are

\footnotetext{
Figure 5 (see following page)

Analysis of new human lipase genes. (a) Schematic representation of the lipase gene cluster on chromosome 10q23.31. Six lipase genes, including the newly described LIPLI (LIPJ), LIPL2 (LIPK), LIPL3 (LIPM) and LIPL4 (LIPN), form a cluster also containing four unrelated genes. (b) Alignment of the protein sequences of the six human lipases from the chromosome $10 q 23.31$ cluster. The amino acids of the catalytic triad are boxed. The alignment was generated with Multalin software [7I]. (c) Hierarchical clustering of human and mouse abhydro-lipase gene family members. The human LIPA, LIPF, LIPK, LIPM and LIPN, but not LIPJ, proteins have clear mouse orthologues (lower-case gene names). The six hypothetical mouse genes found in place of the LIPJ gene (Lipdc l-5) form a separate branch of the phylogenetic tree. This tree was generated with the Tree Top software [72]. Bootstrap values are indicated in red.
} 
(a)

$50 \mathrm{~kb}$

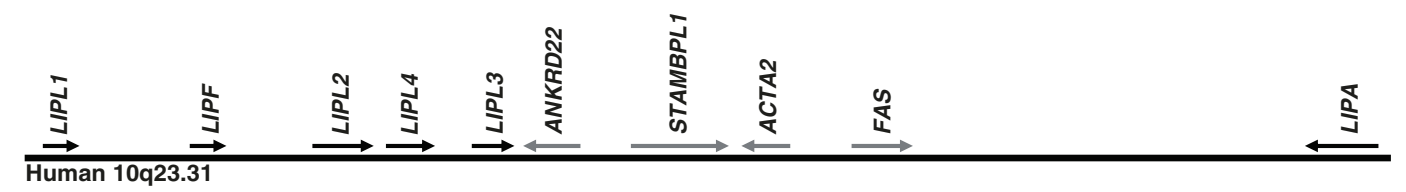

$50 \mathrm{~kb}$

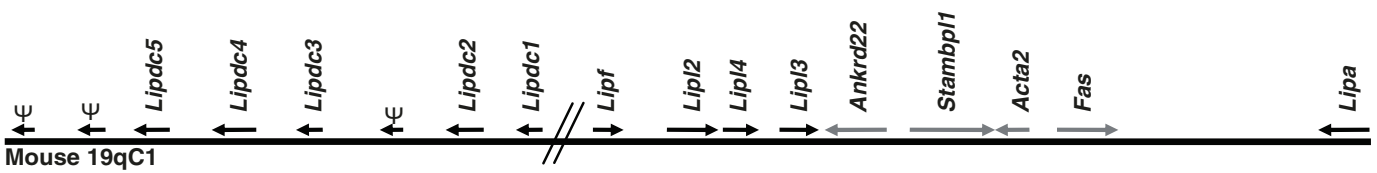

(b)

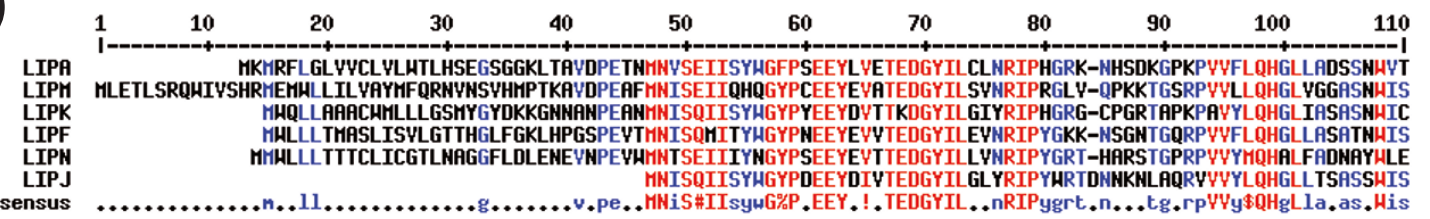

Consensus

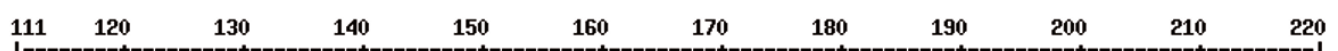

LIPA NLANSSLGFILADAGFDYHMGNSRGTHSRKHKTLSYSQDEFHAFSYDEMAKYDLPASINFILNKTGQEQYY FYGHSGTTIGFIAFSQIPELAKRIKMFFFLGPYASYA

LIPH NLPNNSLGFILADAGFDYMMGNSRGNAHSRKHKTLSIDQDEFHAFSYDEMARFDLPAYINFILQKTGQEKIY YYGYS RGTTMGFIAFSTMPELAQKIKMYFALAPIATYK

作

LIPF NLPNNSLAFTLADAGYDYHLGNSRGNTHARRNLYYSPDSYEFHAFSFDEMAKYDLPATTDFTYKKTGOKQLH FUGHSTGTTIGFTAFSTNPSLAKRIKTFYALAPUATYK

LIPN NYANGSLGFLLADAGYDYMGNSRGNTHSRRHKTLSETDEKFHAFSFDEMAKYDLPGYIDFIYNKTGQEKLY IGHSEGTTIGFYAFSTMPELAQRIKMNFALGPTISFK

LIPJ NLPNNSLGFILADAGYDYMGNSRGNTHSRKHLYLETSSKEFHAFSFDEMAKYDLPASIDFTYKQTRQEEIFYGHS RGTTIGFITFSTISKIAERIKIFFALAPYFSTK

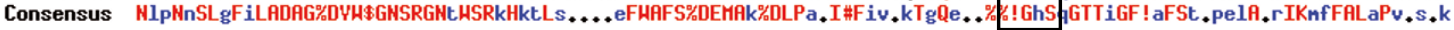
221230
240
250
260
270
280
290
300
310
320
330

LIPA FCTSPMAKLGRLPDHLIKDLFGDKEFLPQSFFLKHLGTHYCTHYILKELCGNLCLLCGFNERNLNHSRYDYYTTHSPFGTSYQNMLHHSQAYKFQKFQAFDHGSSAKNY

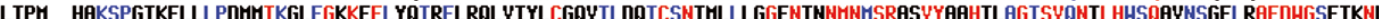

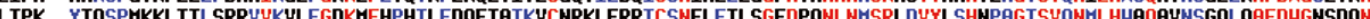

LIPF YTKSLTHKLRFYPQSLFKFIFGDKTFYPHFFDFF

IPU

LIP J YLKSPLTRMTYKUKSTUMPFSGHKDFLPKTSFKKFIGSKLCPLOTFDKTCLNILP

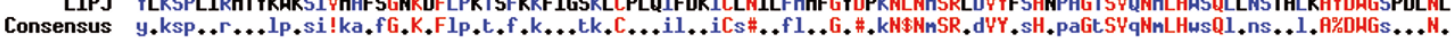

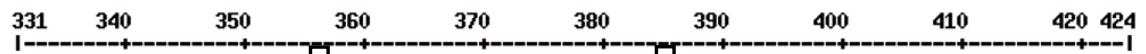

LIPA FHYNQSYPPTYNYKDHLYPTAYHS G G

LIPH EKCNQPTPVR

LIPK MHFHQLTPPLYNITKITEYPTALNGGQ

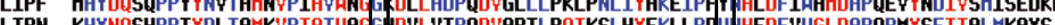

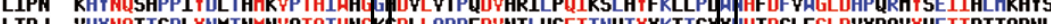

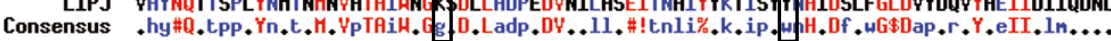

(c)

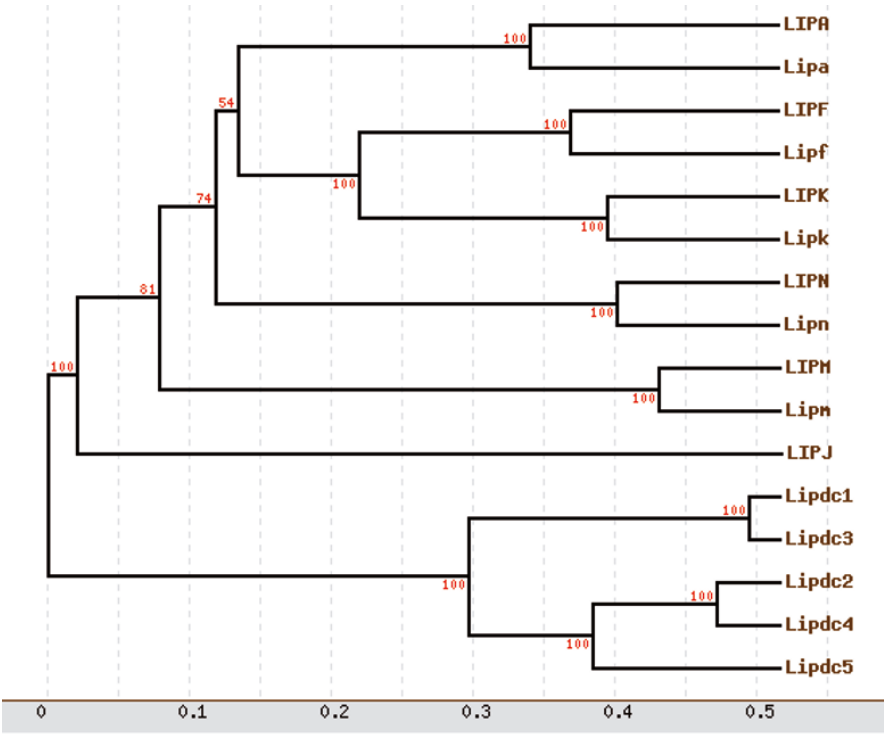

Figure 5 (see legend on previous page) 


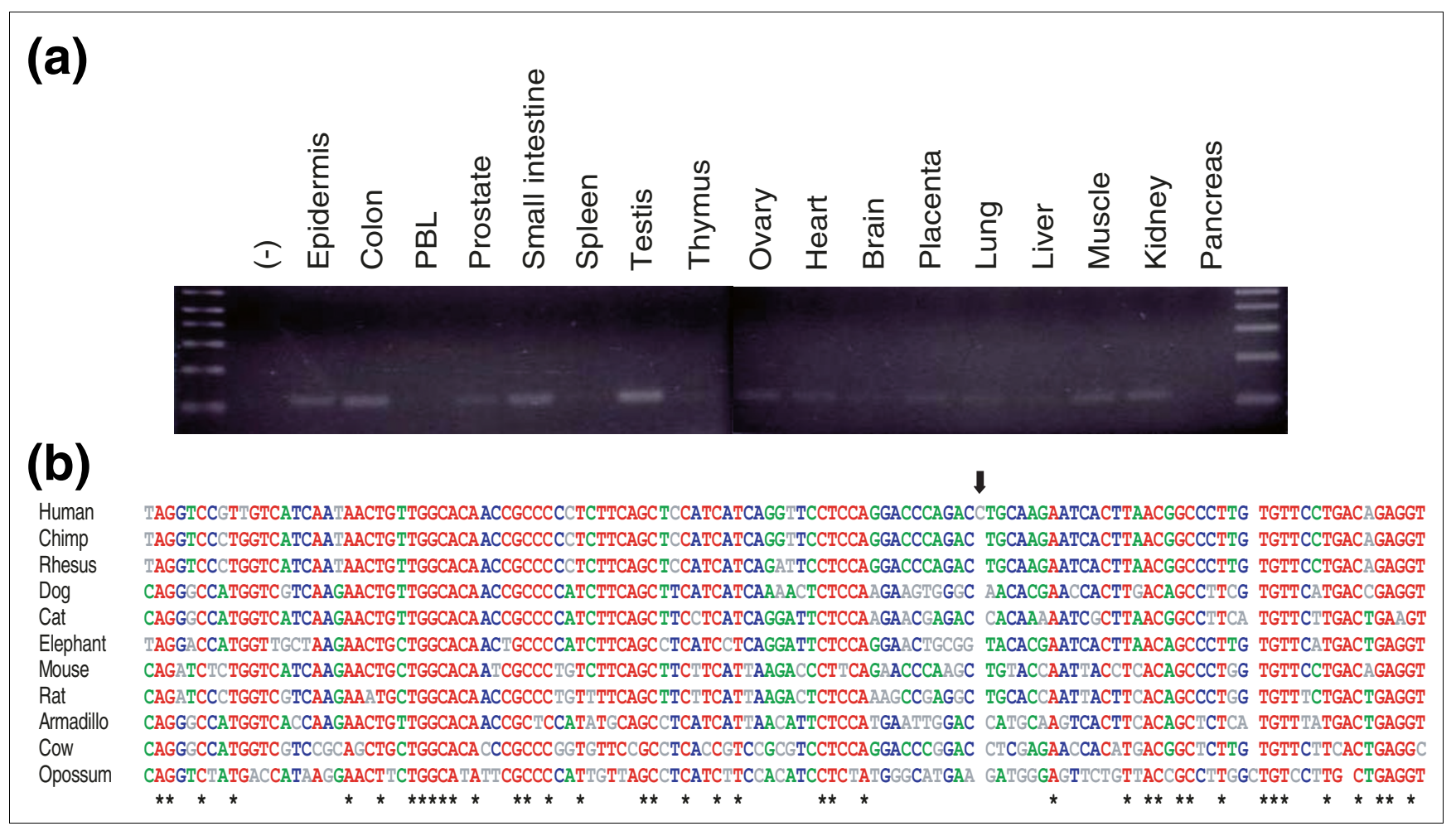

Figure 6

Clorf8I mRNA expression and conservation of the eighth exon among mammals. (a) Expression pattern of the Clorf8I gene. PCR was performed with a commercial panel of CDNAs from 16 human tissues (PBL, peripheral blood leukocytes) and with CDNA prepared from epidermis. The amplified fragment (I20 nt) encompasses exons I3-I4. (b) Sequence alignment of the eighth exon of the Clorf8I gene from II mammals. The sequences were retrieved from the multiz I 7way table of the UCSC Genome Browser [73], and from a BLAST search of the cat genome. The consensus splicing signals are boxed. The black arrow indicates the single nucleotide insertion in the human gene. The alignment was created with Multalin software [7I]. Asterisks indicate the positions conserved in all the sequences. The colors correspond to various levels of consensus, with red for high consensus and grey for low consensus.

responsible for Netherton syndrome (OMIM \#256500), characterized by ichthyosiform erythroderma, bamboo hair and atopic dermatitis. In this framework, we identified three protease inhibitors potentially involved in desquamation.

In addition to the serine protease inhibitor SERPINA12 discussed above, we identified two other members of the serpin superfamily, SERPINB7 and SERPINB12, whose expression in the epidermis is reported here for the first time. Moreover, our real-time PCR experiments clearly show that these two protease inhibitors (as well as SERPINA12) are overexpressed in the uppermost epidermal layers. SERPINB7, also known as Megsin, is deposited in the extracellular matrix by kidney mesangial cells [48], but its targets have not been identified to date. SERPINB12 is expressed in many tissues, and displays inhibitor activity against trypsin-like serine proteases [49]. To understand the roles of these protease inhibitors in desquamation, it is of key interest to determine their molecular targets. Proteases expressed in the skin and potentially involved in desquamation are interesting candidates. Our ORESTES data set includes the serine protease kallikrein 7 (SCCE), which plays a key function in desquamation by cleaving two corneodesmosome components, desmo- collin 1 and corneodesmosin [50]. Surprisingly, we did not detect kallikrein 5 (SCTE), which cleaves another corneodesmosome component, desmoglein 1. However, we detected for the first time the transcription of the $P 11$ gene in the epidermis. This gene encodes a secreted serine protease previously shown to be expressed in the human placenta and various neoplasms of the breast, ovary, testis, and stomach [51]. Its specific targets remain unknown. In epidermis, $P 11$ is upregulated in the granular layer with a $\mathrm{T} 4 / \mathrm{T} 1$ ratio of 35 (Table 7). We suggest that P11 could act in the course of desquamation, either by cleaving corneodesmosome components, or by activating other proteases.

Protease inhibitors might also play a documented role in protecting the body from infection. In addition to the liverexpressed antimicrobial peptide $L E A P-2$, we detected the expression of the $W A P 1$ and $W A P 2$ genes, which encode serine protease inhibitors with antimicrobial activity in mouse tongue and kidney [52]. WAP1 appears to be expressed in all epidermal layers, whereas WAP2 is overexpressed in GKs and could thus play an antimicrobial role in uppermost epidermal layers. Therefore, our study contributes to enlarge the panel of proteases and protease inhibitors potentially involved in 
Table 8

\begin{tabular}{llcl}
\multicolumn{2}{l}{ Monogenic diseases due to mutations of genes involved in lipid metabolism and displaying an epidermal phenotype } \\
\hline Gene & Function & MIM/reference & Pathology \\
\hline$A B C A / 2$ & ABC lipid transporter & $\# 242500$ & Harlequin ichthyosis \\
STS & Steroid sulfatase & +38100 & X-linked ichthyosis \\
GBA & Glucocerebrosidase & $\# 230800$ & Gaucher disease \\
ALOXE3/ALOXBI2 & Arachidonate lipoxygenases & $\# 242100$ & Non-bullous congenital ichthyosiform erythroderma \\
CGI58 (ABHD5) & Putative triglyceride lipase & {$[62]$} & Chanarin-Dorman syndrome \\
LIPH & Phospholipase AI & {$[63]$} & Hair growth defect
\end{tabular}

barrier function, regulation of desquamation and defense against microorganisms.

\section{Genes involved in lipid metabolism}

Mutations of genes involved in various aspects of lipid metabolism are at the origin of several human genodermatoses (Table 8), underlying the key interest in the identification of new, lipid-processing genes expressed in the skin. We identified three new human genes, LIPK, LIPM and LIPN, which encode proteins containing two characteristic domains, the $\alpha / \beta$ hydrolase fold and the abhydrolase associated lipase region. They furthermore contain the consensus pattern of the active domain [53], suggesting that they are bona fide lipase genes (Figure 5b; Additional data file 4). The $L I P K$, $L I P M$ and $L I P N$ genes are strongly specific for the epidermis (Figure 4), and real-time RT-PCR experiments revealed a highly specific expression in GKs, with T4/T1 ratios >100.

Phylogenetic studies showed that the LIPJ, LIPK, LIPM and LIPN proteins are very close to well-characterized members of the family LIPA and LIPF, both also encoded in the 10q23.31 locus. LIPA is a ubiquitous lysosomal cholesterol ester hydrolase (EC 3.1.1.13) [54,55], while LIPF is a secreted triglyceride lipase (EC 3.1.1.3) [56]. Interestingly, both LIPA and LIPF have a low $\mathrm{pH}$ optimum, in agreement with the acidic $\mathrm{pH}$ of the extracellular space in the stratum corneum $[57,58]$.

The extracellular hydrolysis of triglycerides in free fatty acids and glycerol is essential to stratum corneum hydration [59]. Furthermore, long chain free fatty acids represent $16 \%$ of extracellular lipids, cholesterol esters $15 \%$, and free cholesterol 32\% [6o]. Triglyceride lipase enzymatic activity has been detected in the intercellular space of the human stratum corneum [61], and little is known regarding the metabolism and extracellular modifications of cholesterol esters. The LIPK, LIPM and LIPN proteins are most probably secreted and could thus participate in the establishment of the barrier function by catalyzing the maturation of extracellular lipids. The weakly expressed, non-secreted LIPJ protein might play a distinct role.
The $L I P K, L I P M$ and $L I P N$, but not LIPJ, gene orthologues could be identified in several vertebrate species, including mouse and rat. Surprisingly, a cluster of tandem duplicated genes encoding new lipases resides in the mouse and rat genomes, which could eventually increase the lipase repertoire of these species.

The new LIPK, LIPM and LIPN lipide hydrolase genes may play an essential function in lipid metabolism of the most differentiated epidermal layers, and are thus interesting gene candidates for genodermatoses of unknown origin. Accordingly, mutations in the $C G I_{5} 8 / A B H D 5$ gene, which encodes a putative triacylglycerol lipase, are responsible for the Chanarin-Dorman syndrome, a neutral-lipid storage disease with ichthyosis [62]. Mutations in the $L I P H$ gene are responsible for a hair growth defect [63]. Both CGI58/ABHD5 and LIPH proteins resemble the lipases encoded by genes from the 10q23.31 locus as they include an $\alpha / \beta$ hydrolase fold, but they lack the abhydrolase associated lipase region.

In addition to the lipases from the 10q23.31 locus, nine additional genes, predicted to be involved in lipid metabolism, were overexpressed in GKs (Table 8). Their homology with known genes suggests that they might act on fatty acid (PNPLA1, THEM5, ADHD9, FAM83F) or ceramide (LASS3, $\left.A S A H_{3}, S M P D_{3}\right)$ metabolism. Compared to other tissues, lipid metabolism in the epidermis presents many distinct characteristics, as it mainly occurs in the extracellular space. Extracellular lipids play key roles in the barrier function, particularly in hydrophobicity of the skin surface. Our study thus unraveled new actors in this particularly important process, and might shed new light on the etiology of genodermatoses.

\section{Genes of miscellaneous function}

In addition to genes involved in protein degradation and lipid metabolism, we characterized new genes that might be of key importance in skin function. CASZ1 is a transcription factor induced during embryogenesis in the course of mesenchyme differentiation [64]. As its gene targets are presently unknown, its relatively high expression level in the human skin (20 ORESTES) calls for detailed functional studies in this tissue. Finally, eight genes encoding hypothetical proteins without known domains, such as C2Oorf95, CXorf33, or 
LOC440449, also displayed GK-specific expression. Their roles in epidermal differentiation remain completely elusive.

\section{Conclusion}

We have described an original and efficient method for purifying GKs from healthy human epidermis. It is now of key interest to adapt this technique to biopsy fragments from patients suffering from various genodermatoses. The GK is the last cell type in the skin to display transcription activity before cornification, and we describe the expression of 3,387 genes, a proportion of which are expressed in GKs in a highly specific manner. We presume that many of them are important for the establishment of the barrier function, and as such deserve detailed functional studies. Moreover, we provide the scientific community with a list of gene candidates for genodermatoses of unknown origin. In particular, the understanding of complex diseases associated with defects in barrier function, such as psoriasis or atopic dermatitis, might benefit from identification of new epidermis-expressed genes located in associated loci. Among the genes described, some fit with, and even improve, our present knowledge of the barrier function, in particular concerning the fine-tuning of protein degradation and lipid metabolism. The precise function of the corresponding proteins will be assessed using mouse models and immunochemistry methods starting from healthy and pathological human skin. However, many of the genes described herein, often specifically expressed in GKs at high levels, encode putative proteins whose functions are totally obscure but that might well participate in the establishment of the skin barrier. Incidentally, we characterized a new gene, C1orf81, which is specifically inactivated or truncated in humans. Whether this gene loss participated in the establishment of the human species, and thus fits the 'less-is-more' hypothesis [65] remains a fascinating question. Our study of the human GK transcriptome thus opens new avenues for future research in many fields, including the normal functioning of the epidermis, the origin of genodermatoses, and even the emergence of the human species.

\section{Materials and methods Skin samples and RNA extraction}

Normal human skin was obtained from patients undergoing abdominal plastic surgery (kindly provided by Professor JP Chavoin, "Service de Chirurgie Plastique et des Brûlés", Centre Hospitalier Universitaire Rangueil, Toulouse, France) after informed consent and in accordance with Helsinki principles. Subcutaneous fat was promptly removed and strips of skin were incubated, epidermis side up, for $1 \mathrm{~h}$ at $4^{\circ} \mathrm{C}$ in phosphate-buffered saline (PBS) containing $0.5 \mathrm{mg} / \mathrm{ml}$ thermolysin (T7902, Sigma, St Louis, MO, USA). The epidermis was dissected free of dermis tissue with forceps and rinsed in cold PBS. Epidermal fragments were either immediately frozen for total RNA extraction or incubated in $1 \times$ trypsin-EDTA solution (25300-054, Invitrogen, Carlsbad, CA, USA) at $4^{\circ} \mathrm{C}$ under gentle agitation for 15 minutes. The remaining epidermal fragments were rinsed in cold PBS and incubated in another trypsin-EDTA solution, while fetal calf serum (10270098, Invitrogen) was added to the suspended cells (10\% final concentration). After centrifugation, the cells were frozen as dry pellets. The procedure was repeated twice, leading to three successive fractions of dissociated cells named $\mathrm{T} 1$, $\mathrm{T}_{2}$ and $\mathrm{T}_{3}$. The residual fragments ( $\mathrm{T}_{4}$ fraction) were drained on a gauze compress, frozen and ground to a powder under liquid nitrogen. Total RNA was extracted from the various cell batches using the RNeasy extraction kit (Qiagen, Hilden, Germany). Purification of poly(A)+ RNA was always performed from the T4 fraction of individual patients, using oligo(dT)25tagged magnetic beads according to the manufacturer's instructions (Dynal, Oslo, Norway). Two rounds of hybridization to the beads were performed. The mRNAs were treated with DNAse I (Invitrogen) and the absence of genomic DNA was confirmed by PCR using primers for the corneodesmosin gene (GenBank: $\underline{\text { AFO3130). }}$

\section{Morphological analysis of epidermis samples}

After each trypsin incubation, an aliquot of epidermal fragments was fixed in Bouin's solution, embedded in paraffin, and sections $(10 \mu \mathrm{m})$ were stained with hematoxylin-eosin.

\section{Production and analysis of ORESTES}

ORESTES production was essentially performed as described [66]. Purified mRNA (20 ng) was heated 10 minutes at $65^{\circ} \mathrm{C}$, reverse transcribed at $37^{\circ} \mathrm{C}$ for $1 \mathrm{~h}$ with $200 \mathrm{U}$ of Moloney murine leukemia virus reverse transcriptase (Promega, Madison, WI, USA) and $10 \mathrm{pmol}$ of an arbitrary selected primer (18-25 nt) in a final volume of $20 \mu \mathrm{l}$. The reaction products ( 1 $\mu \mathrm{l})$ were amplified by PCR using either the primer used for the reverse transcription, or a single, alternative arbitrary chosen primer. The hybridization step of the first PCR cycle was set at $37^{\circ} \mathrm{C}$, while the 35 remaining cycles were performed in standard conditions with a hybridization temperature complying with the length of the primer (typically $55^{\circ} \mathrm{C}$ ). After gel electrophoresis, products with predominant bands reflecting the amplification of highly abundant sequences were not further processed. Smear-like reaction products were gel-purified with a $500 \mathrm{bp}$ cut-off. Mini-libraries were then produced by T/A cloning of the purified PCR fragments (TOPO-TA cloning kit, Invitrogen).

\section{Plasmid purification and sequencing}

Sequencing was performed by standard procedures (ABI Prism Dye Terminator Cycle Sequencing kit, Applera, Norwalk, CI, USA) after either plasmid purification (Wizard miniprep kit, Promega) or rolling circle amplification of the plasmids.

\section{Sequence analysis}

An automated protocol for the sequence analysis was used to verify sequence quality. Sequences were then analyzed using a stepwise approach. Starting with RepeatMasker [67], 
ORESTES were clustered with the PHRAP algorithm [68]. Consensus sequences or singletons were annotated using BLAST searches against human databases (best-hit among successively RefSeq, Uniprot and EST_Human databases [69]). Sequences were also aligned on the human genome (May 2004 assembly) using BLAT [18] and inserted as a custom track into the UCSC Genome Browser.

\section{Analysis of gene expression}

For quantitative real-time RT-PCR experiments, all primer pairs (available upon request) were chosen to generate amplicons of 100-250 bp encompassing different exons, thus avoiding the amplification of potential contaminating genomic DNA. The primer sequences were designed using Primer3 software [70] and BLAST analysis [69] ensured the absence of similarity to any other human sequence. Reverse transcription was performed by standard procedures, starting from 100 ng of total RNA of each cell batch and using a mixture of oligo(dT) and random hexamers. Amplification assays were performed with the ABI prism 7000 Sequence Detection System and analyzed with the corresponding software (Applied Biosystems, Foster City, CA, USA) using the qPCR ROX-\&GO Green mix (MP Biomedicals, Irvine, CA, USA). Fluorescence was quantified as Ct (threshold cycle) values. Samples were analyzed in triplicate, with differences between the three $\mathrm{Ct}$ values lower than 0.3. Expression levels were calibrated using galectin 7 ( $L G A L S 7$ ), or beta-2-microglubulin (B2M) mRNA as internal controls. The differences between the mean $\mathrm{Ct}$ values of the various amplicons and the reference genes are denoted $(\Delta \mathrm{Ct})$. The difference between $\Delta \mathrm{Ct}$ obtained with the indicated cell samples are labeled $\Delta \Delta \mathrm{Ct}$; $2^{\Delta \Delta C t}$ gave the relative level of gene expression between the T1 and T4 fractions. Control wells containing the SYBR Green PCR master mix and primers without template cDNA emitted no significant fluorescence after 40 cycles.

Human Multiple Tissue cDNA panels I and II obtained from Clontech (Palo Alto, CA, USA) were used as templates for PCR analysis. A control reaction with $\mathrm{T} 4 \mathrm{cDNAs}$ was carried out in parallel. The reactions were conducted for 35 cycles in standard conditions. The PCR products were separated on $1.5 \%$ agarose-TAE gels.

\section{RACE-PCR experiments}

We performed 5' RNA ligase-mediated (RLM)-RACE using the FirstChoice RLM-RACE kit (Ambion, Austin, TX, USA). Briefly, total RNA was dephosphorylated with calf intestine phosphatase then decapped using tobacco acid pyrophosphatase to target full-length mRNA. An adapter was then ligated to mRNA and reverse transcription was performed using random decamers. PCR was performed to amplify the resulting cDNA using the Outer 5' RLM-RACE primer and a gene specific lower primer. Nested PCR was then performed with the Inner 5' RLM-RACE primer. The RACE nested PCR products were cloned into the pCRII-TOPO vector using a TOPO T/A cloning kit (Invitrogen) and sequenced.

\section{NCBI gene ID references}

GAPDH, 2597; SOD1, 6647; ACTB, 60; B2M, 567; HPRT1, 3251; HMBS, 3145; TBP, 6908; UBC, 7316; PNAS-123, 85028; PPP2R5A, 5525; RPS11, 6205; RPS12, 6206; RPL1O, 6134; EIF4A1, 1973; KRTHAP1, 8686.

\section{Additional data files}

The following additional data are available with the online version of this paper. Additional data file 1 is a screen copy of the ORESTES custom track of the human UCSC Genome Browser that shows the genomic localization of the newly described C1orf81 gene with the corresponding ORESTES (chr1:199,164,695-199,202,419; hg18, March 2006). Additional data file 2 is a table of genes (with 100 or less UniGene ESTs) expressed in various tissues but for which epidermal expression had never been assessed. Additional data file 3 is a colored text file containing the alignment of human and rhesus genomic sequences showing the location of the insertion point and tandem site duplication for the hominoid-specific CNOT6L processed retrogene. Additional data file 4 is the alignment of protein sequences of the ten mouse lipases, including Lipdc1-5. The alignment was generated with Multalin software [71]; the amino acids of the catalytic triad are boxed. Additional data file 5 is a table providing gene nomenclature and IDs for five new mouse lipase genes.

\section{Acknowledgements}

Our special thanks go to M-T Ribouchon for excellent technical assistance. We are grateful to $\mathrm{H}$ Brun and $\mathrm{C}$ Offer from the "Service Commun de Séquençage - IFR30" for sequencing the clones EL593304 to EL595248. We thank $D$ Bobermien for her contribution to the production of minilibraries and $M$ Fraisse for histochemistry. This work was supported in part by grants from the "Centre National de la Recherche Scientifique", the Toulouse III Paul Sabatier University, the "Société Française de Dermatologie" and from the "Société de Recherche Dermatologique". Sequencing was funded by the "Consortium National de Recherche en Génomique".

\section{References}

I. Watt FM, Celso CL, Silva-Vargas V: Epidermal stem cells: an update. Curr Opin Genet Dev 2006, 16:518-524.

2. Park GT, Lim SE, Jang SI, Morasso MI: Suprabasin, a novel epidermal differentiation marker and potential cornified envelope precursor. J Biol Chem 2002, 277:45 I95-45202.

3. Oomizu S, Sahuc F, Asahina K, Inamatsu M, Matsuzaki T, Sasaki M, Obara M, Yoshizato K: Kdap, a novel gene associated with the stratification of the epithelium. Gene 2000, 256:19-27.

4. Makino T, Takaishi M, Morohashi M, Huh NH: Hornerin, a novel profilaggrin-like protein and differentiation-specific marker isolated from mouse skin. J Biol Chem 200I, 276:47445-47452.

5. Lee WH, Jang S, Lee JS, Lee Y, Seo EY, You KH, Lee SC, Nam KI, Kim JM, Kee SH, et al:: Molecular cloning and expression of human keratinocyte proline-rich protein (hKPRP), an epidermal marker isolated from calcium-induced differentiating keratinocytes. J Invest Dermatol 2005, I 25:995-1000.

6. Kalinin AE, Kajava AV, Steinert PM: Epithelial barrier function: assembly and structural features of the cornified cell envelope. Bioessays 2002, 24:789-800.

7. Elias PM, Cullander C, Mauro T, Rassner U, Komuves L, Brown BE, Menon GK: The secretory granular cell: the outermost granular cell as a specialized secretory cell. J Investig Dermatol Symp Proc 1998, 3:87-100.

8. Guerrin M, Simon M, Montezin M, Haftek M, Vincent C, Serre G: 
Expression cloning of human corneodesmosin proves its identity with the product of the $S$ gene and allows improved characterization of its processing during keratinocyte differentiation. I Biol Chem 1998, 273:22640-22647.

9. Dias Neto E, Correa RG, Verjovski-Almeida S, Briones MR, Nagai MA, da Silva W Jr, Zago MA, Bordin S, Costa FF, Goldman GH, et al.: Shotgun sequencing of the human transcriptome with ORF expressed sequence tags. Proc Natl Acad Sci USA 2000, 97:349I-3496.

10. Vandesompele J, De Preter K, Pattyn F, Poppe B, Van Roy N, De Paepe A, Speleman F: Accurate normalization of real-time quantitative RT-PCR data by geometric averaging of multiple internal control genes. Genome Biol 2002, 3:RESEARCH0034.

II. Bonnet-Duquennoy M, Abaibou H, Tailhardat M, Lazou K, Bosset S, Le Varlet B, Cleuziat $P$, Kurfurst R: Study of housekeeping gene expression in human keratinocytes using OLISAtrade mark, a long-oligonucleotide microarray and qRT-PCR. Eur J Dermatol 2006, 16:136-140.

12. Magnaldo T, Fowlis D, Darmon M: Galectin-7, a marker of all types of stratified epithelia. Differentiation 1998, 63:159-168.

13. Fisher C, Angus B, Rees J: In-situ hybridization using digoxigenin-labelled probes in human skin. BrJ Dermatol I991, 125:516-520.

14. Sondell $\mathrm{B}$, Jonsson $\mathrm{M}$, Dyberg $\mathrm{P}$, Egelrud $\mathrm{T}$ : In situ evidence that the population of Langerhans cells in normal human epidermis may be heterogeneous. Br J Dermatol 1997, 136:687-693.

15. Matsui T, Hayashi-Kisumi F, Kinoshita Y, Katahira S, Morita K, Miyachi $\mathrm{Y}$, Ono $\mathrm{Y}$, Imai T, Tanigawa $\mathrm{Y}$, Komiya $\mathrm{T}$, et al.: Identification of novel keratinocyte-secreted peptides dermokine-alpha/beta and a new stratified epithelium-secreted protein gene complex on human chromosome 19q13.1. Genomics 2004, 84:384-397.

16. Moffatt P, Salois P, St-Amant N, Gaumond MH, Lanctot C: Identification of a conserved cluster of skin-specific genes encoding secreted proteins. Gene 2004, 334:|23-I3|.

17. Toulza E, Galliano MF, Jonca N, Gallinaro H, Mechin MC, IshidaYamamoto A, Serre G, Guerrin M: The human dermokine gene: description of novel isoforms with different tissue-specific expression and subcellular location. J Invest Dermatol 2006, 126:503-506.

18. Kent WJ: BLAT - the BLAST-like alignment tool. Genome Res 2002, I 2:656-664.

19. UCSC Custom Track: ORESTES from Granular Keratinocytes of Human Epidermis [http://genome.ucsc.edu/cgi-bin/ hgTracks?org=human\&hgt.customText=http://udear.cnrs.free.fr/ orestes.txt]

20. Brandner JM, Kief S, Wladykowski E, Houdek P, Moll I: Tight junction proteins in the skin. Skin Pharmacol Physiol 2006, 19:7I-77.

21. Furuse M, Hata M, Furuse K, Yoshida Y, Haratake A, Sugitani Y, Noda T, Kubo A, Tsukita S: Claudin-based tight junctions are crucial for the mammalian epidermal barrier: a lesson from claudinI-deficient mice. / Cell Biol 2002, I 56: 1099-IIIII.

22. Vinckenbosch N, Dupanloup I, Kaessmann H: Evolutionary fate of retroposed gene copies in the human genome. Proc Natl Acad Sci USA 2006, 103:3220-3225.

23. Rogers MS, Kobayashi T, Pittelkow MR, Strehler EE: Human calmodulin-like protein is an epithelial-specific protein regulated during keratinocyte differentiation. Exp Cell Res 200I, 267:216-224.

24. The snoRNABase [http://www-snorna.biotoul.fr/]

25. Weber MJ: Mammalian small nucleolar RNAs are mobile genetic elements. PLoS Genet 2006, 2:e205.

26. Smith CM, Steitz JA: Classification of gas5 as a multi-smallnucleolar-RNA (snoRNA) host gene and a member of the 5'terminal oligopyrimidine gene family reveals common features of snoRNA host genes. Mol Cell Biol 1998, 18:6897-6909.

27. Ji P, Diederichs S, Wang W, Boing S, Metzger R, Schneider PM, Tidow $\mathrm{N}$, Brandt $B$, Buerger $\mathrm{H}$, Bulk E, et al.: MALAT-I, a novel noncoding RNA, and thymosin beta 4 predict metastasis and survival in early-stage non-small cell lung cancer. Oncogene 2003, 22:803|-804|.

28. Geirsson A, Paliwal I, Lynch RJ, Bothwell AL, Hammond GL: Class II transactivator promoter activity is suppressed through regulation by a trophoblast noncoding RNA. Transplantation 2003 , 76:387-394.

29. Zhang $Y$, Tycko B: Monoallelic expression of the human $\mathbf{H} \mathbf{9}$ gene. Nat Genet 1992, I:40-44.
30. Thorvaldsen JL, Duran KL, Bartolomei MS: Deletion of the HI9 differentially methylated domain results in loss of imprinted expression of HI9 and Igf2. Genes Dev 1998, I 2:3693-3702.

3I. Blakytny R, Jude EB, Martin Gibson J, Boulton AJ, Ferguson MW: Lack of insulin-like growth factor I (IGFI) in the basal keratinocyte layer of diabetic skin and diabetic foot ulcers. J Pathol 2000, 190:589-594.

32. Bennett WR, Crew TE, Slack JM, Ward A: Structural-proliferative units and organ growth: effects of insulin-like growth factor 2 on the growth of colon and skin. Development 2003, 130:1079-1088.

33. Katayama S, Tomaru Y, Kasukawa T, Waki K, Nakanishi M, Nakamura M, Nishida H, Yap CC, Suzuki M, Kawai J, et al:: Antisense transcription in the mammalian transcriptome. Science 2005, 309: I564-I566.

34. Strausberg RL, Feingold EA, Grouse LH, Derge JG, Klausner RD, Collins FS, Wagner L, Shenmen CM, Schuler GD, Altschul SF, et al.: Generation and initial analysis of more than 15,000 full-length human and mouse cDNA sequences. Proc Natl Acad Sci USA 2002, 99:16899-16903.

35. Kraut J: Serine proteases: structure and mechanism of catalysis. Annu Rev Biochem 1977, 46:33I-358.

36. Brzozowski AM, Derewenda U, Derewenda ZS, Dodson GG, Lawson DM, Turkenburg JP, Bjorkling F, Huge-Jensen B, Patkar SA, Thim L: A model for interfacial activation in lipases from the structure of a fungal lipase-inhibitor complex. Nature 199|, 35 I:49| -494.

37. Smith TP, Grosse WM, Freking BA, Roberts AJ, Stone RT, Casas E, Wray JE, White J, Cho J, Fahrenkrug SC, et al.: Sequence evaluation of four pooled-tissue normalized bovine cDNA libraries and construction of a gene index for cattle. Genome Res 200I, I I:626-630.

38. Hida K, Wada J, Eguchi J, Zhang H, Baba M, Seida A, Hashimoto I, Okada T, Yasuhara A, Nakatsuka A, et al.: Visceral adipose tissuederived serine protease inhibitor: a unique insulin-sensitizing adipocytokine in obesity. Proc Natl Acad Sci USA 2005, 102:10610-10615.

39. Singh G, Lykke-Andersen J: New insights into the formation of active nonsense-mediated decay complexes. Trends Biochem Sci 2003, 28:464-466.

40. Matsufuji S, Matsufuji T, Miyazaki Y, Murakami Y, Atkins JF, Gesteland RF, Hayashi S: Autoregulatory frameshifting in decoding mammalian ornithine decarboxylase antizyme. Cell 1995, 80:5I-60.

4I. Hahn Y, Lee B: Identification of nine human-specific frameshift mutations by comparative analysis of the human and the chimpanzee genome sequences. Bioinformatics 2005, 2 I (Suppl I):il $186-194$.

42. Wang $X$, Grus WE, Zhang J: Gene losses during human origins. PLoS Biol 2006, 4:e52.

43. Winter H, Langbein L, Krawczak M, Cooper DN, Jave-Suarez LF, Rogers MA, Praetzel S, Heidt PJ, Schweizer J: Human type I hair keratin pseudogene phihHaA has functional orthologs in the chimpanzee and gorilla: evidence for recent inactivation of the human gene after the Pan-Homo divergence. Hum Genet 200I, 108:37-42.

44. Hahn $Y$, Lee $B$ : Human-specific nonsense mutations identified by genome sequence comparisons. Hum Genet 2006, I 19:169-178.

45. Marques AC, Dupanloup I, Vinckenbosch N, Reymond A, Kaessmann $\mathrm{H}$ : Emergence of young human genes after a burst of retroposition in primates. PLoS Biol 2005, 3:e357.

46. Zeeuwen PL: Epidermal differentiation: the role of proteases and their inhibitors. Eur J Cell Biol 2004, 83:761-773.

47. Zeeuwen PL, van Vlijmen-Willems IM, Hendriks W, Merkx GF, Schalkwijk J: A null mutation in the cystatin M/E gene of ichq mice causes juvenile lethality and defects in epidermal cornification. Hum Mol Genet 2002, I I:2867-2875.

48. Miyata $T$, Inagi $R$, Nangaku M, Imasawa $T$, Sato $M$, Izuhara $Y$, Suzuki $D$, Yoshino A, Onogi H, Kimura M, et al.: Overexpression of the serpin megsin induces progressive mesangial cell proliferation and expansion. J Clin Invest 2002, 109:585-593.

49. Askew YS, Pak SC, Luke CJ, Askew DJ, Cataltepe S, Mills DR, Kato H, Lehoczky J, Dewar K, Birren B, et al.: SERPINB I 2 is a novel member of the human ov-serpin family that is widely expressed and inhibits trypsin-like serine proteinases. J Biol Chem 200I, 276:49320-49330.

50. Caubet C, Jonca N, Lopez F, Esteve JP, Simon M, Serre G: Homooligomerization of human corneodesmosin is mediated by its N-terminal glycine loop domain. J Invest Dermatol 2004, 
I 22:747-754.

5I. Grundmann U, Romisch J, Siebold B, Bohn H, Amann E: Cloning and expression of a cDNA encoding human placental protein I I, a putative serine protease with diagnostic significance as a tumor marker. DNA Cell Biol 1990, 9:243-250.

52. Hagiwara K, Kikuchi T, Endo Y, Huqun, Usui K, Takahashi M, Shibata N, Kusakabe T, Xin H, Hoshi S, et al.: Mouse SWAMI and SWAM2 are antibacterial proteins composed of a single whey acidic protein motif. J Immunol 2003, I 70:1973-1979.

53. Cousin X, Hotelier T, Giles K, Lievin P, Toutant JP, Chatonnet A: The alpha/beta fold family of proteins database and the cholinesterase gene server ESTHER. Nucleic Acids Res 1997, 25:143-146.

54. Du H, Witte DP, Grabowski GA: Tissue and cellular specific expression of murine lysosomal acid lipase mRNA and protein. J Lipid Res 1996, 37:937-949.

55. Anderson RA, Sando GN: Cloning and expression of cDNA encoding human lysosomal acid lipase/cholesteryl ester hydrolase. Similarities to gastric and lingual lipases. J Biol Chem I99I, 266:22479-22484.

56. Bodmer MW, Angal S, Yarranton GT, Harris TJ, Lyons A, King DJ, Pieroni G, Riviere C, Verger R, Lowe PA: Molecular cloning of a human gastric lipase and expression of the enzyme in yeast. Biochim Biophys Acta 1987, 909:237-244.

57. Ohman $H$, Vahlquist $A$ : In vivo studies concerning a $\mathbf{p H}$ gradient in human stratum corneum and upper epidermis. Acta Derm Venereol 1994, 74:375-379.

58. Hanson KM, Behne MJ, Barry NP, Mauro TM, Gratton E, Clegg RM: Two-photon fluorescence lifetime imaging of the skin stratum corneum pH gradient. Biophys J 2002, 83: 1682-1690.

59. Fluhr JW, Mao-Qiang M, Brown BE, Wertz PW, Crumrine D, Sundberg JP, Feingold KR, Elias PM: Glycerol regulates stratum corneum hydration in sebaceous gland deficient (asebia) mice. J Invest Dermatol 2003, 120:728-737.

60. Norlen L, Nicander I, Lundh Rozell B, Ollmar S, Forslind B: Interand intra-individual differences in human stratum corneum lipid content related to physical parameters of skin barrier function in vivo. J Invest Dermatol 1999, I I 2:72-77.

61. Menon GK, Grayson S, Elias PM: Cytochemical and biochemical localization of lipase and sphingomyelinase activity in mammalian epidermis. J Invest Dermatol 1986, 86:59I-597.

62. Lefevre C, Jobard F, Caux F, Bouadjar B, Karaduman A, Heilig R, Lakhdar H, Wollenberg A, Verret JL, Weissenbach J, et al.: Mutations in CGI-58, the gene encoding a new protein of the esterase/ lipase/thioesterase subfamily, in Chanarin-Dorfman syndrome. Am J Hum Genet 2001, 69:1002-1012.

63. Kazantseva A, Goltsov A, Zinchenko R, Grigorenko AP, Abrukova AV, Moliaka YK, Kirillov AG, Guo Z, Lyle S, Ginter EK, et al.: Human hair growth deficiency is linked to a genetic defect in the phospholipase gene LIPH. Science 2006, 3 I 4:982-985.

64. Liu Z, Yang X, Tan F, Cullion K, Thiele CJ: Molecular cloning and characterization of human Castor, a novel human gene upregulated during cell differentiation. Biochem Biophys Res Commun 2006, 344:834-844.

65. Olson MV: When less is more: gene loss as an engine of evolutionary change. Am J Hum Genet 1999, 64:18-23.

66. Leerkes MR, Caballero OL, Mackay A, Torloni H, O'Hare MJ, Simpson AJ, de Souza SJ: In silico comparison of the transcriptomederived from purified normal breast cells and breast tumor cell lines reveals candidate upregulated genes in breast tumor cells. Genomics 2002, 79:257-265.

67. RepeatMasker Open-3.0 [http://www.repeatmasker.org]

68. PHRAP Software [http://www.phrap.org/]

69. Altschul SF, Madden TL, Schaffer AA, Zhang J, Zhang Z, Miller W, Lipman DJ: Gapped BLAST and PSI-BLAST: a new generation of protein database search programs. Nucleic Acids Res 1997, 25:3389-3402.

70. Rozen S, Skaletsky H: Primer3 on the WWW for general users and for biologist programmers. Methods Mol Biol 2000, I 32:365-386.

71. Corpet F: Multiple sequence alignment with hierarchical clustering. Nucleic Acids Res 1988, I 6:1088I-10890.

72. TreeTop: Phylogenetic Tree Prediction [http:// www.genebee.msu.su/services/phtree_reduced.html]

73. Blanchette M, Kent WJ, Riemer C, Elnitski L, Smit AF, Roskin KM, Baertsch R, Rosenbloom K, Clawson H, Green ED, et al.: Aligning multiple genomic sequences with the threaded blockset aligner. Genome Res 2004, I4:708-7I5. 